2010

\title{
Effects of Multitemperature Nonequilibrium on Compressible Homogeneous Turbulence
}

Wei Liao

Old Dominion University

Yan Peng

Old Dominion University, ypeng@odu.edu

Li-Shi Luo

Old Dominion University, lluo@odu.edu

Follow this and additional works at: https://digitalcommons.odu.edu/mathstat_fac_pubs

Part of the Fluid Dynamics Commons, Mathematics Commons, and the Statistical, Nonlinear, and Soft Matter Physics Commons

\section{Repository Citation}

Liao, Wei; Peng, Yan; and Luo, Li-Shi, "Effects of Multitemperature Nonequilibrium on Compressible Homogeneous Turbulence" (2010). Mathematics \& Statistics Faculty Publications. 11.

https://digitalcommons.odu.edu/mathstat_fac_pubs/11

\section{Original Publication Citation}

Liao, W., Peng, Y., \& Luo, L. S. (2010). Effects of multitemperature nonequilibrium on compressible homogeneous turbulence. Physical Review E, 81(4), 046704. doi:10.1103/PhysRevE.81.046704 


\title{
Effects of multitemperature nonequilibrium on compressible homogeneous turbulence
}

\author{
Wei Liao, ${ }^{*}$ Yan Peng, ${ }^{\dagger}$ and Li-Shi Luo ${ }^{\ddagger}$ \\ Department of Mathematics \& Statistics and Center for Computational Sciences, Old Dominion University, Norfolk, Virginia 23529, USA
}

(Received 25 February 2010; published 28 April 2010)

\begin{abstract}
We study the effects of the rotational-translational energy exchange on the compressible decaying homogeneous isotropic turbulence (DHIT) in three dimensions through direct numerical simulations. We use the gas-kinetic scheme coupled with multitemperature nonequilibrium based on the Jeans-Landau-Teller model. We investigate the effects of the relaxation time of rotational temperature, $Z_{R}$, and the initial ratio of the rotational and translational temperatures, $T_{R 0} / T_{L 0}$, on the dynamics of various turbulence statistics including the kinetic energy $K(t)$, the dissipation rate $\varepsilon(t)$, the energy spectrum $E(k, t)$, the root mean square of the velocity divergence $\theta^{\prime}(t)$, the skewness $S_{u}(t)$ and the flatness $F_{u}(t)$ of the velocity derivatives, and the probability distribution functions of the local Mach number Ma and the shocklet strength $\chi$. The larger the $Z_{R}$ is, the faster the compressibility decays after an initial time. Similarly, with a fixed $T_{L 0}$, the higher the initial energy ratio $T_{R 0} / T_{L 0}$, the weaker is the compressibility in the flow. It is also observed that the effect of $T_{R 0} / T_{L 0}$ is strong in all times in the decay, while the effect of $Z_{R}$ is severe only in the later times passing through the stage with strong nonlinearity. We also observe that the multitemperature model does not affect the self-similarities obeyed by the probability distribution functions of $\mathrm{Ma}$ and $\chi$, which appear to be a robust feature of the compressible DHIT.
\end{abstract}

DOI: 10.1103/PhysRevE.81.046704

PACS number(s): 47.11.-j, 47.40.-x, 94.05.Lk, 47.45.Ab

\section{INTRODUCTION}

Hypersonic flows are often associated with nonthermodynamic-equilibrium (NTE) conditions [1-3]. Obviously, turbulence in hypersonic flows under NTE conditions is far more challenging than its incompressible counterpart because the Kolmogorov paradigm, which is the theoretical basis of incompressible turbulence, may not be valid any longer for the compressible turbulence under NTE conditions. Due to a growing interest in hypersonics in recent years, direct numerical simulations (DNSs) have been increasingly used as a tool to study compressible turbulence [4-11]. It is hoped that a comparison of DNS and experimental data can provide a guide for modeling of compressible turbulence.

Under NTE conditions, the internal degrees of freedom of gas molecules are excited and they play an important role in the physics of compressible flows. Within the framework of hydrodynamics, the effects due to the internal degrees of freedom are modeled through the bulk viscosity or a variable ratio of specific heats $\gamma(T):=c_{p} / c_{v}$ (cf., e.g., [2]). Another approach is to introduce multiple temperatures to represent internal degrees of freedom and the energy equations for internal degrees of freedom, which are coupled to the Navier-Stokes equations, are solved simultaneously with the hydrodynamic equations (cf., e.g., [2,12]). The relaxation process of internal degrees of freedom has to be modeled in this approach and one of the most well-known models is the Jeans-Landau-Teller (JLT) model [13-16]. In spite of its limitations (e.g., [17]), the JLT model remains as a very popular model due to its simplicity. In the present work, we

\footnotetext{
*wliao@odu.edu

†ypeng@odu.edu

"1luo@odu.edu
}

will model nonequilibrium compressible flows with the JLT multitemperature model.

There are very few studies about the effects of multitemperature models on compressible turbulent flows. Bertolotti studied the influence of rotational and vibrational energy relaxations on the stability of the supersonic boundary layer [18] and found the main effect of the rotational relaxation is the damping high-frequency instability. Grigor'ev et al. studied the effect of vibrational relaxation on the nonlinear interaction of a vortex perturbation in a shear flow of a highly nonequilibrium diatomic gas [19]. El Morsli and Proulx incorporated the multitemperature thermal and chemical nonequilibrium into a renormalization-group (RNG)-based $k-\epsilon$ turbulence model for a turbulent flow in a supersonic nozzle coupled with a plasma torch [3]. To our knowledge, the effect of multitemperature models on compressible homogeneous isotropic turbulence has not yet to be studied.

The objective of this work is to quantitatively investigate the effects of the multitemperature model on the compressible decaying homogeneous isotropic turbulence. We will use the multitemperature gas-kinetic scheme (GKS) to carry out the DNS of the compressible decaying homogeneous isotropic turbulence (DHIT), which has been a subject of continuing interest $[5,20-23]$. This work is a sequel to our previous work [24], in which we study the numerics of the GKS and evaluate their effects on the compressible DHIT. The GKS method is a finite-volume (FV) method derived from the linearized Boltzmann equation [24-33], of which the Bhatnagar-Gross-Krook (BGK) model equation [34] is a special case. The fluxes in the GKS method are reconstructed from the approximated particle-velocity distribution function $f(\boldsymbol{x}, \boldsymbol{\xi}, t)$ in phase space $\Gamma:=(\boldsymbol{x}, \boldsymbol{\xi})$. Owing to its kinetic nature, the GKS method can be extended to simulate nonequilibrium flows, which are beyond the validity of the NavierStokes equations, such as the Burnett flow $[35,36]$ and shock structures in gases $[32,33,37,38]$. The multitemperature model has already been incorporated into the GKS method to 
simulate various nonequilibrium flows including shock structures in gases $[39,40]$, near-continuum flows [41,42], and hypersonic nonequilibrium flows [43].

The remainder of the paper is organized as follows. In Sec. II, we will describe in details the formulations of the GKS methods for three-dimensional (3D) compressible flows with multitemperature nonequilibrium and provide a discussion of the macroscopic equations modeled by the GKS method with multitemperature nonequilibrium. In Sec. III, we will discuss succinctly the compressible decaying homogeneous isotropic turbulence and the turbulence statistics to be computed. In Sec. IV, we present our results. We investigate the effects of the multitemperature nonequilibrium on various turbulence statistics, such as the total energy, the dissipation rate, the energy spectrum, the skewness, the flatness, and the probability distribution functions of the local Mach number and the shocklet strength. Finally, we conclude the paper with a summary in Sec. V.

\section{NUMERICAL METHODS}

The GKS for fully compressible Navier-Stokes equations has been discussed extensively in our previous work $[24,32,44,45]$. In this work, we will consider the GKS with the Jeans-Landau-Teller multitemperature model for nonequilibrium flows. The details of flux reconstruction, if not discussed here, can be found in our earlier work [24].

\section{A. Gas kinetic scheme with multitemperature nonequilibrium}

The starting point of gas-kinetic schemes is the linearized Boltzmann equation (e.g., [46])

$$
\partial_{t} f+\boldsymbol{\xi} \cdot \nabla f=\mathcal{L}(f, f),
$$

where $f:=f(\boldsymbol{x}, \boldsymbol{\xi}, \boldsymbol{\zeta}, t)$ is the single-particle distribution function of space $\boldsymbol{x}$, particle velocity $\boldsymbol{\xi}$, particle internal degrees of freedom $\zeta$ of dimension $Z$, and time $t ; \mathcal{L}$ is the linearized collision operator. For the sake of simplicity and without losing generality in the context of the linearized Boltzmann equation, we will use the BGK single relaxation-time model for $\mathcal{L}[34]$

$$
\partial_{t} f+\boldsymbol{\xi} \cdot \nabla f=-\frac{1}{\tau}\left[f-f^{(0)}\right],
$$

where $\tau$ is the relaxation time toward its equilibrium. To consider nonequilibrium effects due to internal degrees of freedom of gas molecules, the rotational temperature $T_{R}$ is introduced, in addition to the translational temperature $T_{L}$. Thus, the Maxwellian equilibrium distribution function $f^{(0)}$ must also include $T_{R}$,

$$
f^{(0)}=\rho\left(\frac{\beta_{L}}{2 \pi}\right)^{D / 2}\left(\frac{\beta_{R}}{2 \pi}\right)^{Z / 2} e^{-1 / 2\left(\beta_{L} c \cdot c+\beta_{R} \zeta \zeta\right)},
$$

where $\boldsymbol{c}:=(\boldsymbol{\xi}-\boldsymbol{u})$ is the peculiar velocity, $R$ is the gas constant, $\rho$ and $\boldsymbol{u}$ are the density and flow, respectively, $\beta_{L}$ $=\left(R T_{L}\right)^{-1}$, and $\beta_{R}=\left(R T_{R}\right)^{-1}$. By integrating along the characteristics [47], one can obtain the following solution of the BGK Eq. (2):

$$
f(\boldsymbol{x}+\boldsymbol{\xi} t, t)=e^{-t / \tau} f_{0}+\frac{1}{\tau} \int_{0}^{t} f^{(0)}\left(\boldsymbol{x}^{\prime}, \boldsymbol{\xi}, \boldsymbol{\zeta}, t^{\prime}\right) e^{\left(t^{\prime}-t\right) / \tau} d t^{\prime},
$$

where $\boldsymbol{x}^{\prime}:=\boldsymbol{x}+\boldsymbol{\xi} t^{\prime}$ and the initial state $f_{0}:=f(\boldsymbol{x}, \boldsymbol{\xi}, \boldsymbol{\zeta}, t=0)$. The GKS is formulated based on the above equation. With $f_{0}$ and $f_{0}^{(0)}:=f^{(0)}(\boldsymbol{x}, \boldsymbol{\xi}, \boldsymbol{\zeta}, t=0)$ given, one can construct an approximate solution for $f$ at a later time $t>0$.

For the sake of convenience, we shall use the following notations for the vectors of $(D+2)$ dimensions:

$$
\begin{gathered}
\boldsymbol{\Psi}:=\left[1, \boldsymbol{\xi},\left(\xi^{2}+\zeta^{2}\right) / 2, \zeta^{2} / 2\right]^{\dagger}, \\
\mathbf{W}:=\left(\rho, \rho \boldsymbol{u}, \rho E, \rho e_{R}\right)^{\dagger}=\int f \boldsymbol{\Psi} d \Xi, \\
\mathbf{F}_{\alpha}:=\int f \boldsymbol{\Psi} \xi_{\alpha} d \Xi, \quad \alpha \in\{x, y, z\}:=\{1,2,3\}, \\
\mathbf{h}:=\left(\rho, \boldsymbol{u}, T_{L}, T_{R}\right)^{\dagger}, \\
\mathbf{h}^{\prime}:=\left(\rho^{-1}, \beta_{L} \boldsymbol{u},\left(\beta_{L} c^{2}-D\right) / 2 T_{L},\left(\beta_{R} \zeta^{2}-Z\right) / 2 T_{R}\right)^{\dagger},
\end{gathered}
$$

where $\dagger$ denotes the transpose operator, $\boldsymbol{\Psi}, \mathbf{W}, \mathbf{F}_{\alpha}$, and $\mathbf{h}$ are the $(D+2)$-dimensional vectors whose components are the collisional invariants, the flow variables, the fluxes along the $\alpha$ axis, and the primitive variables, respectively, and $\Xi$ $:=(\xi, \zeta)$ denotes the single-particle velocity space and the internal degrees of freedom. The flow variables, $\rho, \rho \boldsymbol{u}, \rho E$, and $\rho e_{R}$, are the density, the momentum, the total energy, and the rotational energy, respectively, and they are the moments of $f$. Among the flow variables, $\rho, \rho \boldsymbol{u}$, and $\rho E$ are the conserved quantities, while $\rho e_{R}$ is not. The specific total energy can be decomposed into the specific internal energy $e$ $:=R\left(D T_{L}+Z T_{R}\right) / 2$ and the specific kinetic energy $u^{2} / 2$, i.e., $E=e+u^{2} / 2$; the specific rotational energy is given by $e_{R}$ $=Z R T_{R} / 2$. The total number of internal degrees of freedom is $Z=(5-3 \gamma) /(\gamma-1)$ and $\gamma=c_{p} / c_{v}$ is the ratio of specific heat.

Based on the Chapman-Enskog analysis, the collision does not alter the values of the conserved variables $\rho, \rho \boldsymbol{u}$, and $\rho E$. Because the internal energy $\rho e_{R}$ is not a conserved quantity, it is not an invariant during the collision. Therefore, the collision term in Eq. (2) can be written as

$$
-\frac{1}{\tau} \int\left(f-f^{(0)}\right) \boldsymbol{\Psi} d \Xi=\mathbf{\Xi}=(0,0,0,0,0, s)^{\dagger},
$$

where the source term $s$ is introduced to account for the effect due to the rotational mode. We will use the relaxation model for the rotational energy $e_{R}$ based on the JeansLandau-Teller model [13-16] for internal degrees of freedom $[12,39,41,43]$,

$$
s=-\frac{1}{Z_{R} \tau} \rho\left(e_{R}-e_{R}^{*}\right),
$$

where the parameter $Z_{R}$ is introduced to overcome the deficiency of the BGK model with one single relaxation parameter $\tau$ which determines all the transport coefficients in the model and 


$$
\begin{gathered}
e_{R}^{*}=\frac{1}{2} Z R T_{R}^{*}, \\
T_{R}^{*}=\frac{1}{(D+Z)}\left(D T_{L}+Z T_{R}\right)=T .
\end{gathered}
$$

Since the advection operator in the Boltzmann equation is linear, i.e., $\boldsymbol{\xi} \cdot \boldsymbol{\nabla} f=\boldsymbol{\nabla} \cdot(\boldsymbol{\xi} f)$, operator splitting among $D$ dimensions can be applied without approximation to affect its multidimensional nature. Thus we can show one the construction of the GKS in the $x$ direction and fluxes along other directions can be easily duplicated. We denote a cell center by $\boldsymbol{x}_{i, j, k}$ and its left and right cell boundaries in the $x$ coordinate by $\boldsymbol{x}_{i-1 / 2, j, k}$ and $\boldsymbol{x}_{i+1 / 2, j, k}$, respectively. For simplicity, we set the initial time $t_{0}=0$, so that the solution (4) at position $\boldsymbol{x}_{i+1 / 2, j, k}$ and time $t$ is

$$
\begin{aligned}
f\left(\boldsymbol{x}_{i+1 / 2, j, k}, t\right)= & e^{-t / \tau} f_{0}\left(\boldsymbol{x}_{i+1 / 2, j, k}-\boldsymbol{\xi} t\right) \\
& +\frac{1}{\tau} \int_{0}^{t} f^{(0)}\left(\boldsymbol{x}^{\prime}, t^{\prime}\right) e^{-\left(t-t^{\prime}\right) / \tau} d t^{\prime}
\end{aligned}
$$

where $\boldsymbol{x}^{\prime}:=\boldsymbol{x}_{i+1 / 2, j, k}-\boldsymbol{\xi}\left(t-t^{\prime}\right)$ is the coordinate of the particle trajectory. In the above equation, we omitted the variables in $f$ which remain unchanged in time. Initially, only the values of the hydrodynamic variables, $\rho, \rho \boldsymbol{u}, \rho E$, and $\rho e_{R}$ are given at the cell center $\boldsymbol{x}_{i, j, k}$, but the fluxes are to be evaluated at the cell boundaries $\boldsymbol{x}_{i \pm 1 / 2, j, k}=0$. Therefore, both $f_{0}$ and $f^{(0)}\left(x^{\prime}, t^{\prime}\right)$ in the above equation are to be constructed from the hydrodynamic variables through the Boltzmann equation and Taylor expansions of $f$.

The BGK equation (2) can be formally written as

$$
f=f^{(0)}-\tau d_{t} f, \quad d_{t}:=\partial_{t}+\boldsymbol{\xi} \cdot \boldsymbol{\nabla} .
$$

For the purpose of simulating the Navier-Stokes equations, we can use an approximated $f$ with $f=f^{(0)}-\tau d_{t} f^{(0)}$. Consequently, the initial value $f_{0}(\boldsymbol{x}, 0)$ can be approximated as

$$
\begin{aligned}
f_{0}(\boldsymbol{x}, 0) & \approx\left[1-\tau\left(\partial_{t}+\boldsymbol{\xi} \cdot \boldsymbol{\nabla}\right)\right] f^{(0)}(\boldsymbol{x}, 0) \\
& =\left[1-\boldsymbol{h}^{\prime} \cdot\left(\partial_{t}+\boldsymbol{\xi} \cdot \boldsymbol{\nabla}\right) \mathbf{h}\right] f^{(0)}(\boldsymbol{x}, 0) .
\end{aligned}
$$

In addition, the equilibrium can be expanded in a Taylor series about $\boldsymbol{x}_{i+1 / 2, j, k}=0$,

$$
\begin{aligned}
f^{(0)}(\boldsymbol{x}, 0) & \approx[1+\boldsymbol{x} \cdot \boldsymbol{\nabla}] f^{(0)}(0,0) \\
& =\left[1+\mathbf{h}^{\prime} \cdot(\boldsymbol{x} \cdot \boldsymbol{\nabla}) \mathbf{h}\right] f^{(0)}(0,0),
\end{aligned}
$$

where $\boldsymbol{x}:=(x, y, z)$. By substituting Eq. (12) into Eq. (11), we have

$$
\begin{aligned}
f_{0}(\boldsymbol{x}, 0) & \approx\left[1+\mathbf{h}^{\prime} \cdot(\boldsymbol{x} \cdot \boldsymbol{\nabla}) \mathbf{h}\right]\left[1-\boldsymbol{h}^{\prime} \cdot\left(\partial_{t}+\boldsymbol{\xi} \cdot \boldsymbol{\nabla}\right) \mathbf{h}\right] f^{(0)}(0,0) \\
& =[1+\boldsymbol{a} \cdot(\boldsymbol{x}-\boldsymbol{\xi} \tau)-A \tau] f^{(0)}(0,0),
\end{aligned}
$$

where $\boldsymbol{a}:=\left(a_{1}, a_{2}, a_{3}\right):=\left(\mathbf{h}^{\prime} \cdot \partial_{x} \mathbf{h}, \mathbf{h}^{\prime} \cdot \partial_{y} \mathbf{h}, \mathbf{h}^{\prime} \cdot \partial_{z} \mathbf{h}\right)$ and $A$ $=\mathbf{h}^{\prime} \cdot \partial_{t} \mathbf{h}$ are functions of $\boldsymbol{\xi}, \boldsymbol{\zeta}$, and the hydrodynamic variables $\rho, \boldsymbol{u}, T_{L}$, and $T_{R}$ and their gradients. They are related by the following compatibility condition:

$$
\int f^{(n)} \mathbf{\Psi} d \Xi=\mathbf{0}, \quad \forall \quad n>0,
$$

where $f^{(n)}$ is the $n$th order Chapman-Enskog expansion of $f$ and $f^{(0)}$ is the Maxwellian of Eq. (12). Therefore, the firstorder compatibility condition

$$
\begin{aligned}
\int f^{(1)} \boldsymbol{\Psi} d \Xi & =-\tau \int d_{t} f^{(0)} \boldsymbol{\Psi} d \Xi \\
& =-\tau \int(A+\boldsymbol{a} \cdot \boldsymbol{\xi}) f^{(0)} \mathbf{\Psi} d \Xi=0
\end{aligned}
$$

leads to the relation between $A$ and $\boldsymbol{a}:=\left(a_{1}, a_{2}, a_{3}\right)$,

$$
\int A f^{(0)} \boldsymbol{\Psi} d \boldsymbol{\Xi}=-\int \boldsymbol{a} \cdot \boldsymbol{\xi} f^{(0)} \boldsymbol{\Psi} d \boldsymbol{\Xi} .
$$

We can concisely write the end results of $\boldsymbol{a}=\boldsymbol{\nabla} \ln f^{(0)}$ and $A=\partial_{t} \ln f^{(0)}$ following a similar derivation for a monatomic gas given by [48]

$$
\begin{aligned}
\boldsymbol{a}= & \boldsymbol{\nabla} \ln \rho+\left(\frac{c^{2}}{2 R T_{L}}-\frac{D}{2}\right) \boldsymbol{\nabla} \ln T_{L} \\
& +\left(\frac{\zeta^{2}}{2 R T_{R}}-\frac{Z}{2}\right) \boldsymbol{\nabla} \ln T_{R}+\frac{1}{R T} \sum_{\alpha=1}^{3} c_{\alpha} \nabla u_{\alpha}, \\
A= & -\boldsymbol{a} \cdot \boldsymbol{\xi}+\left(\frac{c^{2}}{2 R T_{L}}-\frac{D+Z}{2}\right) \boldsymbol{c} \cdot \boldsymbol{\nabla} \ln T_{L} \\
& +\left(\frac{\zeta^{2}}{2 R T_{R}}-\frac{Z}{2}\right) \boldsymbol{c} \cdot \boldsymbol{\nabla} \ln T_{R}+\frac{1}{R T}\left(\boldsymbol{c} \boldsymbol{c}-\frac{1}{3} c^{2} \mathrm{I}\right): \boldsymbol{\nabla} \boldsymbol{u},
\end{aligned}
$$

where $\boldsymbol{c}:=(\boldsymbol{\xi}-\boldsymbol{u})$ is the peculiar velocity, $c^{2}:=\boldsymbol{c} \cdot \boldsymbol{c}$, and $\mathrm{I}$ is the $3 \times 3$ identity matrix. For fully compressible flows, the variables in the conservation form $\left(\rho, \rho \boldsymbol{u}, \rho E, \rho e_{R}\right)$ are used instead of the primitive ones $\left(\rho, \boldsymbol{u}, T_{L}, T_{R}\right)$. The Jacobians between the two sets of variables are readily available to convert one set of variables to the other. When computing the gradients $\partial_{\alpha} \mathbf{h}$ for the coefficients $\boldsymbol{a}:=\left(a_{1}, a_{2}, a_{3}\right)$ in Eq. (13), we allow the hydrodynamic variables to be discontinuous at the cell boundary $\boldsymbol{x}_{i \pm 1 / 2, j, k}$, if appropriate.

As for $f^{(0)}(\boldsymbol{x}, t)$ in the integrand of Eq. (9), it can be evaluated by its Taylor expansion,

$$
\begin{aligned}
f^{(0)}(\boldsymbol{x}, t) & \approx\left(1+t \partial_{t}+\boldsymbol{x} \cdot \boldsymbol{\nabla}\right) f^{(0)}(0,0) \\
& =f^{(0)}(0,0)\left[1+\mathbf{h}^{\prime} \cdot\left(t \partial_{t}+\boldsymbol{x} \cdot \boldsymbol{\nabla}\right) \mathbf{h}\right] \\
& =(1+\overline{\boldsymbol{a}} \cdot \boldsymbol{x}+\bar{A} t) f^{(0)}(0,0),
\end{aligned}
$$

where $\overline{\boldsymbol{a}}:=\left(\bar{a}_{1}, \bar{a}_{2}, \bar{a}_{3}\right)$ and $\bar{A}$ are similar to $\boldsymbol{a}:=\left(a_{1}, a_{2}, a_{3}\right)$ and $A$, respectively. The difference between $\boldsymbol{a}$ and $\overline{\boldsymbol{a}}$ is that in $\boldsymbol{a}$ $:=\left(a_{1}, a_{2}, a_{3}\right)$, both the hydrodynamic variables $\left(\rho, \boldsymbol{u}, T_{L}, T_{R}\right)$ and their gradients $\boldsymbol{\nabla}\left(\rho, \boldsymbol{u}, T_{L}, T_{R}\right)$ are allowed to be discontinuous, while in $\overline{\boldsymbol{a}}:=\left(\bar{a}_{1}, \bar{a}_{2}, \bar{a}_{3}\right)$, the hydrodynamic variables are assumed to be continuous, but their gradients in the direction normal to cell interfaces are not [24].

In this work, we will restrict our focus on the compressible flows which do not require a limiter and artificial dissi- 
pations to stabilize the code. For such flows, we can assume that hydrodynamic variables and their gradients are continuous across cell boundaries. Consequently, we have $\boldsymbol{a}=\overline{\boldsymbol{a}}$ and $A=\bar{A}$. By substituting Eqs. (13) and (17) into Eq. (9), we can obtain the distribution function at a cell interface as follows $[45,48,49]$ :

$$
f\left(\boldsymbol{x}_{i+1 / 2, j, k}, t\right)=f_{0}^{(0)}[1-\tau(\boldsymbol{a} \cdot \boldsymbol{\xi}+A)+A t] .
$$

In the above formula, interpolations used to compute the macroscopic flow variables and their gradients at the cell boundaries are determined by the accuracy required. To attain second-order accuracy, we can use the following linear interpolations for the flow variables and their gradients around cell boundaries $(i+1 / 2)$ :

$$
\begin{gathered}
\mathbf{W}_{i+1 / 2, j, k}=\frac{1}{2}\left(\mathbf{W}_{i, j, k}+\mathbf{W}_{i+1, j, k}\right), \\
\partial_{x} \mathbf{W}_{i+1 / 2, j, k}=\frac{1}{\Delta x}\left(\mathbf{W}_{i+1, j, k}-\mathbf{W}_{i, j, k}\right), \\
\partial_{y} \mathbf{W}_{i+1 / 2, j, k}=\frac{1}{2 \Delta y}\left(\mathbf{W}_{i+1 / 2, j+1, k}-\mathbf{W}_{i+1 / 2, j-1, k}\right), \\
\partial_{z} \mathbf{W}_{i+1 / 2, j, k}=\frac{1}{2 \Delta z}\left(\mathbf{W}_{i+1 / 2, j, k+1}-\mathbf{W}_{i+1 / 2, j, k-1}\right) .
\end{gathered}
$$

We can also use the following interpolations to achieve thirdorder accuracy:

$$
\begin{aligned}
\mathbf{W}_{i+1 / 2, j, k}=\frac{9}{16}\left(\mathbf{W}_{i, j, k}+\mathbf{W}_{i+1, j, k}\right)-\frac{1}{16}\left(\mathbf{W}_{i-1, j, k}+\mathbf{W}_{i+2, j, k}\right), \\
\partial_{x} \mathbf{W}_{i+1 / 2, j, k}=\frac{5}{4 \Delta x}\left(\mathbf{W}_{i+1, j, k}-\mathbf{W}_{i, j, k}\right) \\
-\frac{1}{12 \Delta x}\left(\mathbf{W}_{i+2, j, k}-\mathbf{W}_{i-1, j, k}\right), \\
\partial_{y} \mathbf{W}_{i+1 / 2, j, k}=\frac{8}{12 \Delta y}\left(\mathbf{W}_{i+1 / 2, j+1, k}-\mathbf{W}_{i+1 / 2, j-1, k}\right) \\
-\frac{1}{12 \Delta y}\left(\mathbf{W}_{i+1 / 2, j+2, k}-\mathbf{W}_{i+1 / 2, j-2, k}\right), \quad(20) \\
\partial_{z} \mathbf{W}_{i+1 / 2, j, k}=\frac{8}{12 \Delta z}\left(\mathbf{W}_{i+1 / 2, j, k+1}-\mathbf{W}_{i+1 / 2, j, k-1}\right) \\
-\frac{1}{12 \Delta z}\left(\mathbf{W}_{i+1 / 2, j, k+2}-\mathbf{W}_{i+1 / 2, j, k-2}\right) . \quad(20
\end{aligned}
$$

Note that, in the above constructions for the gradients of flow variables, all the values of the flow variables at the cell boundaries $(i+1 / 2)$ must be interpolated from the cellaveraged values at the cell centers.

The relaxation time $\tau$ in Eq. (18) is determined by the local macroscopic flow variables through

$$
\tau=\mu / p
$$

where $\mu$ is the dynamic viscosity and $p$ is the pressure. For continuous flows considered here, the value of the dynamic viscosity $\mu\left(\boldsymbol{x}_{i+1 / 2, j, k}\right)$ in Eq. (21) is determined by

$$
\mu=\left.\mu_{0}\left(T / T_{0}\right)^{0.76}\right|_{x_{i+1 / 2, j, k}},
$$

where $\mu_{0}$ and $T_{0}$ are material-dependent constants and $T$ $:=\left(D T_{L}+Z T_{R}\right) /(D+Z)$. Here, we use the arithmetic average of $T_{L}$ and $T_{R}$ to compute the viscosity. This choice of $T$ in Eq. (22) is not unique because one can use either $T=T_{L}$, or $T=\sqrt{T_{L} T_{R}}$, or other more general expressions (e.g., [50] and references therein). We have tested with $T=T_{L}$ in Eq. (22) and find that the choice of the expression for $T$ has little effect on the turbulence statistics because the two temperatures equilibrate very fast in the hydrodynamic time scale, as we will see later in Sec. IV A. In Eq. (21), the pressure $p$ is only related to the translational temperature through $p$ $=\rho R T_{L}$. Equations (21) and (22) use the hydrodynamic variables $\mathbf{h}\left(\boldsymbol{x}_{i+1 / 2, j, k}\right)$ at the previous time step $t=t_{n-1}$, which can be obtained through $\mathbf{W}\left(\boldsymbol{x}_{i+1 / 2, j, k}\right)$ given by Eq. (18) or (20).

With $f$ given at the cell boundaries, the time-dependent fluxes can be computed

$$
\mathbf{F}_{x}^{i+1 / 2, j, k}=\int \xi_{\alpha} \Psi f\left(\boldsymbol{x}_{i+1 / 2, j, k}, t\right) d \Xi .
$$

Therefore, the fluxes $\mathbf{F}_{\alpha}, \alpha \in\{x, y, z\}$, are fully by determined the approximated distribution function $f$ at the cell interface $\boldsymbol{x}_{i+1 / 2, j, k}$. By integrating $\mathbf{F}_{\alpha}$ of Eq. (23) over one time step $\Delta t$, we obtain the total fluxes

$$
\begin{aligned}
& \overline{\mathbf{F}}_{x}^{i \pm 1 / 2, j, k}=\int_{0}^{\Delta t} \mathbf{F}_{x}^{i \pm 1 / 2, j, k} d t, \\
& \overline{\mathbf{F}}_{y}^{i, j \pm 1 / 2, k}=\int_{0}^{\Delta t} \mathbf{F}_{y}^{i, j \pm 1 / 2, k} d t, \\
& \overline{\mathbf{F}}_{z}^{i, j, k \pm 1 / 2}=\int_{0}^{\Delta t} \mathbf{F}_{z}^{i, j, k \pm 1 / 2} d t .
\end{aligned}
$$

The governing equations in the finite-volume formulation can then be written as

$$
\begin{aligned}
\mathbf{W}_{i j k}^{n+1}= & \mathbf{W}_{i j k}^{n}-\frac{1}{\Delta x}\left(\overline{\mathbf{F}}_{x}^{i+1 / 2, j, k}-\overline{\mathbf{F}}_{x}^{i-1 / 2, j, k}\right) \\
& -\frac{1}{\Delta y}\left(\overline{\mathbf{F}}_{y}^{i, j+1 / 2, k}-\overline{\mathbf{F}}_{y}^{i, j-1 / 2, k}\right) \\
& -\frac{1}{\Delta z}\left(\overline{\mathbf{F}}_{z}^{i, j, k+1 / 2}-\overline{\mathbf{F}}_{z}^{i, j, k-1 / 2}\right)+\mathbf{S}_{i j k}^{n} \Delta t,
\end{aligned}
$$

where $\mathbf{S}_{i j k}^{n}$ is the source term for the rotational energy relaxation given by Eqs. (6) and (7). The above equations are used to update the flow variables $\rho, \rho \boldsymbol{u}, \rho E$, and $\rho e_{R}$.

For the GKS to be stable, the time step size $\Delta t$ must satisfy the Courant-Friedrichs-Lewy (CFL) conditions 

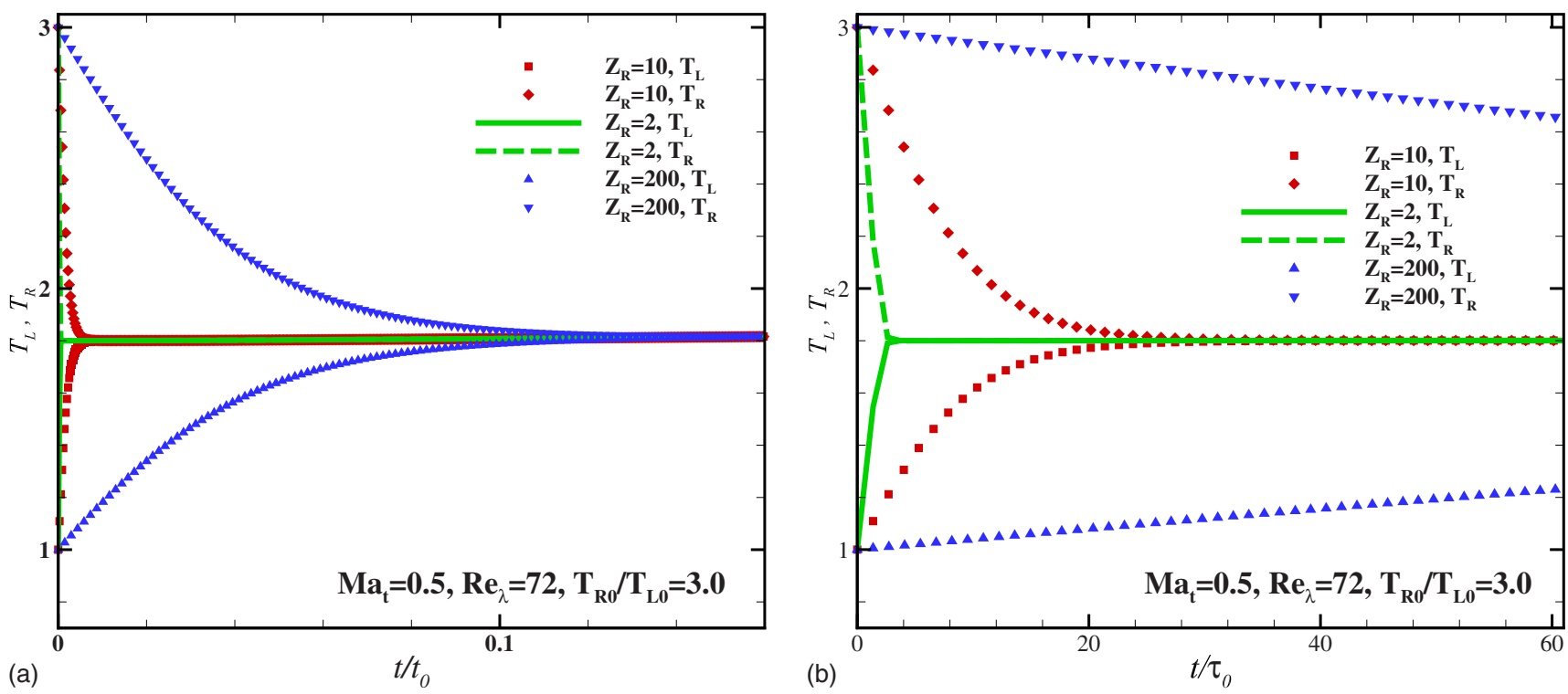

FIG. 1. (Color online) $Z_{R}$ dependence of the translational temperature $T_{L}$ and rotational temperature $T_{R} \cdot \mathrm{Ma}_{t}=0.5, \operatorname{Re}_{\lambda}=72, N^{3}=128^{3}$. Temperatures as functions of two different dimensionless times $t / t_{0}$ (left) and $t / \tau_{0}$ (right).

$$
\Delta t \leq \frac{\Delta x \mu_{\mathrm{CFL}}}{\left(|\boldsymbol{u}|+c_{s}\right)\left(1+2 / \operatorname{Re}^{*}\right)},
$$

where $\mu_{\mathrm{CFL}}$ is the CFL number, $c_{s}:=\sqrt{\gamma R T_{L}}$ is the speed of sound, $|\boldsymbol{u}|$ is the magnitude of the macroflow velocity $\boldsymbol{u}$, and $\operatorname{Re}^{*}:=|\boldsymbol{u}| \Delta x / \nu$ is the grid Reynolds number.

To correct the unity Prandtl number due to the BGK model [51], we can replace the coefficient $\tau$ in the heat flux $\boldsymbol{q}$ by the appropriate coefficient determined by the Prandtl number Pr in the total-energy flux $\boldsymbol{K}[26,32,33]$

$$
\begin{gathered}
\boldsymbol{K}^{\text {new }}=\boldsymbol{K}+\left(\frac{1}{\operatorname{Pr}}-1\right) \boldsymbol{q}, \\
\boldsymbol{K}:=\frac{1}{2} \int\left(\xi^{2}+\zeta^{2}\right) \boldsymbol{\xi} f d \boldsymbol{\Xi}, \\
\boldsymbol{q}:=\frac{1}{2} \int\left(c^{2}+\zeta^{2}\right) \boldsymbol{c} f d \boldsymbol{\Xi},
\end{gathered}
$$

where $\boldsymbol{q}$ is the time-dependent heat flux. With the smooth distribution function $f$ given by Eq. (18), the heat flux $\boldsymbol{q}$ can be computed as the following $[26,32,33]$ :

$$
\boldsymbol{q}=-\tau \int \frac{1}{2}\left(c^{2}+\zeta^{2}\right) \boldsymbol{c} f_{0}^{(0)}(\boldsymbol{a} \cdot \boldsymbol{\xi}+A) d \boldsymbol{\Xi},
$$

where $\boldsymbol{c}:=(\boldsymbol{\xi}-\boldsymbol{u})$ and $\boldsymbol{u}$ is the flow velocity evaluated at the cell interface at time $t=0$. By using Eq. (27a) with $\boldsymbol{q}$ given by Eq. (28), we can adjust the Prandtl number freely and arbitrarily. Because all the moments needed in Eq. (28) have been computed in the evaluation of the total-energy flux $\boldsymbol{K}$, thus additional effort required to compute $\boldsymbol{K}^{\text {new }}$ is negligible.

\section{B. Macroscopic equations}

The macroscopic equations solved by the GKS presented in the previous section are

$$
\begin{gathered}
\partial_{t} \rho+\boldsymbol{\nabla} \cdot \boldsymbol{\rho} \boldsymbol{u}=0, \\
\partial_{t} \rho \boldsymbol{u}+\boldsymbol{\nabla} \cdot \boldsymbol{\rho} \boldsymbol{u} \boldsymbol{u}+\boldsymbol{\nabla} p=\boldsymbol{\nabla} \cdot \boldsymbol{\sigma}, \\
\partial_{t} \rho E+\boldsymbol{\nabla} \cdot(\rho E \boldsymbol{u})+\boldsymbol{\nabla} \cdot(p \boldsymbol{u})=\boldsymbol{\nabla} \cdot \boldsymbol{q}+\boldsymbol{\nabla} \cdot(\boldsymbol{\sigma} \cdot \boldsymbol{u}), \\
\partial_{t} \rho e_{R}+\boldsymbol{\nabla} \cdot\left(\rho e_{R} \boldsymbol{u}\right)=\boldsymbol{\nabla} \cdot \boldsymbol{q}_{R}+\boldsymbol{\nabla} \cdot\left(\sigma_{R} \boldsymbol{u}\right)+s, \\
\sigma_{i j}:=\mu\left(\partial_{i} u_{j}+\partial_{j} u_{i}\right)-\frac{2}{3} \delta_{i j}\left(\mu \boldsymbol{\nabla} \cdot \boldsymbol{u}-\sigma_{R}\right), \\
\sigma_{R}:=\frac{3 \rho R}{2} \frac{Z\left(T_{R}-T_{L}\right)}{Z_{R}(D+Z)}, \\
\boldsymbol{q}:=\frac{1}{2} \mu R \boldsymbol{v}\left[(D+2) T_{L}+Z T_{R}\right], \\
\boldsymbol{q}_{R}:=\frac{1}{2} \mu Z R \boldsymbol{\nabla} T_{R},
\end{gathered}
$$

where $s$ is the source term to model the energy exchange between the rotational and translational modes, defined by Eq. (7). In the limit of an infinitesimal departure from the equilibrium state, i.e., $\left(e_{R}-e_{R}^{*}\right) \rightarrow 0$, the rotational energy Eq. (29d) leads to the following relationship [43]:

$$
T_{R}-T_{L} \approx \frac{2}{3} \tau Z_{R} T \nabla \cdot \boldsymbol{u}
$$

and consequently 

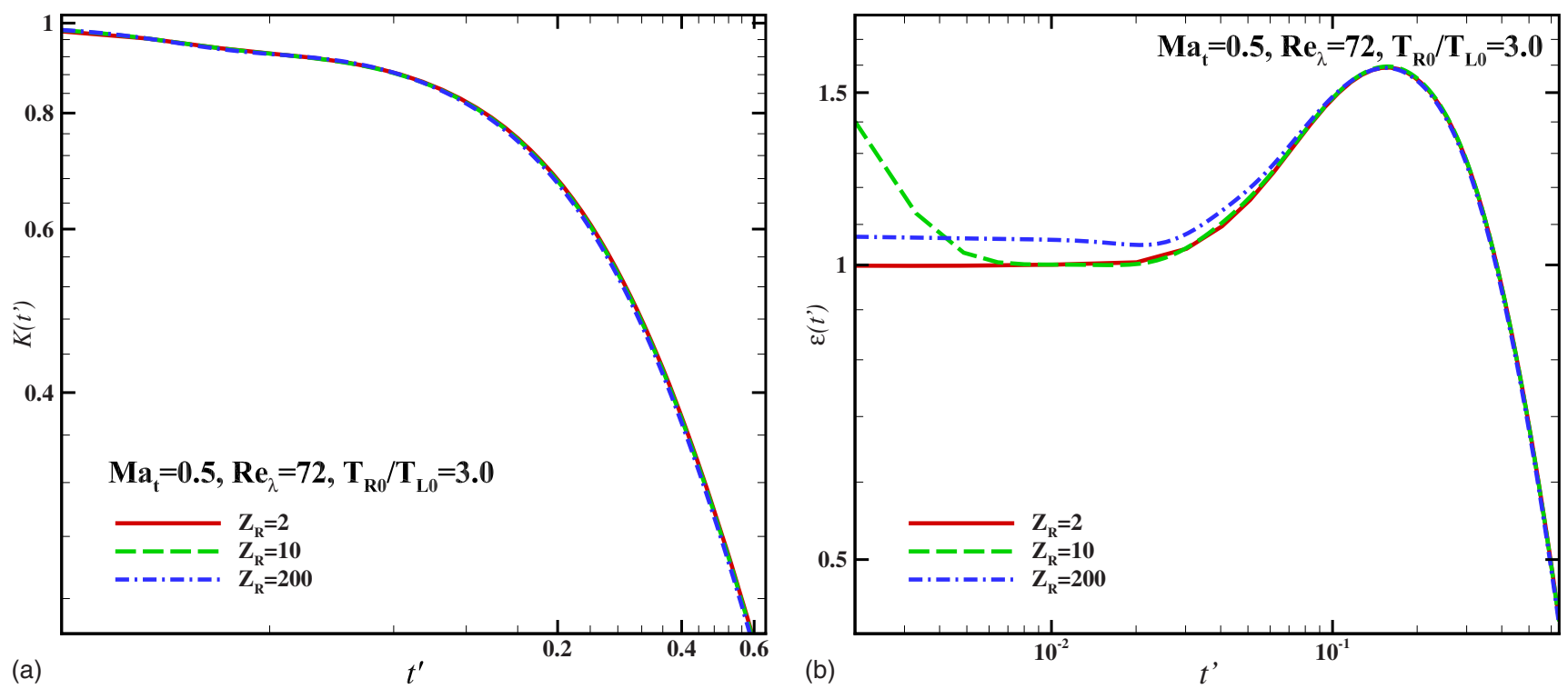

FIG. 2. (Color online) $Z_{R}$ dependence of the kinetic energy $K(t) / K_{0}$ (left) and dissipation rate $\varepsilon(t) / \varepsilon_{0}(\operatorname{right}) . \mathrm{Ma}_{t}=0.5, \mathrm{Re}_{\lambda}=72$, $N^{3}=128^{3}$.

$$
\sigma_{R} \approx \frac{Z}{(D+Z)} \mu \boldsymbol{\nabla} \cdot \boldsymbol{u}=\frac{3}{2} \eta \nabla \cdot \boldsymbol{u},
$$

where $\eta$ is the bulk viscosity. Then, the macroscopic flow equations (29) reduce to the compressible Navier-Stokes equations with the usual translational temperature $T=T_{L}$,

$$
\partial_{t} \rho+\nabla \cdot \rho \boldsymbol{u}=0
$$

$$
\partial_{t} \rho \boldsymbol{u}+\boldsymbol{\nabla} \cdot \boldsymbol{\rho u} \boldsymbol{u}+\boldsymbol{\nabla} p=\boldsymbol{\nabla} \cdot \boldsymbol{\sigma},
$$

$$
\partial_{t} \rho E+\nabla \cdot \rho E \boldsymbol{u}+\boldsymbol{\nabla} \cdot p \boldsymbol{u}=\boldsymbol{\nabla} \cdot \boldsymbol{q}+\boldsymbol{\nabla} \cdot(\boldsymbol{\sigma} \cdot \boldsymbol{u}),
$$

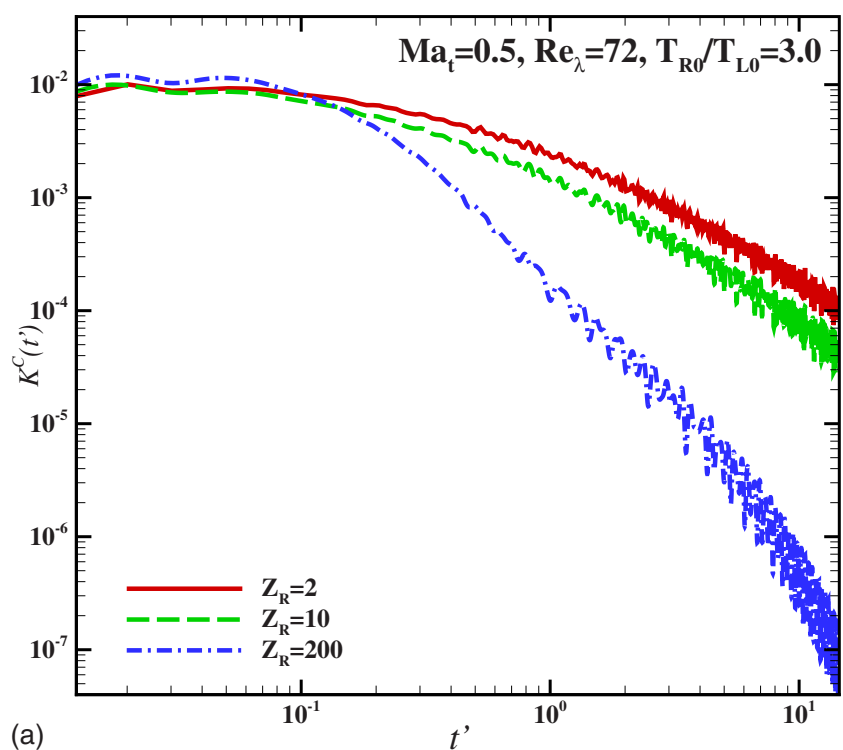

$$
\sigma_{i j}:=\mu\left(\partial_{i} u_{j}+\partial_{j} u_{i}\right)+\left(\eta-\frac{2}{3} \mu\right) \delta_{i j} \nabla \cdot \boldsymbol{u},
$$

$$
\boldsymbol{q}:=\kappa \nabla T
$$

where $\kappa=c_{p} \mu / \operatorname{Pr}$ is the heat conductivity and the bulk viscosity is given by

$$
\eta=\frac{2 Z}{3(D+Z)} \mu
$$

For $D=3$ and $Z=2$, then $\eta=4 \mu / 15$.

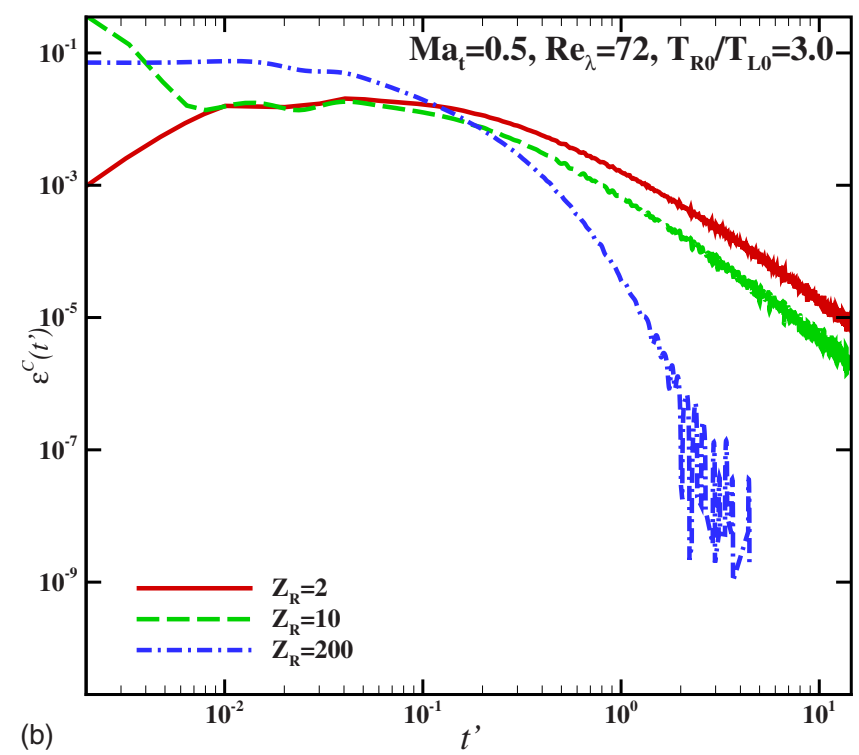

FIG. 3. (Color online) $Z_{R}$ dependence of the dilatational components of the kinetic energy, $K^{C}(t) / K_{0}$, (left) and the dissipation rate, $\varepsilon^{C}(t) / \varepsilon_{0}$, (right). $\mathrm{Ma}_{t}=0.5, \operatorname{Re}_{\lambda}=72, N^{3}=128^{3}$. 

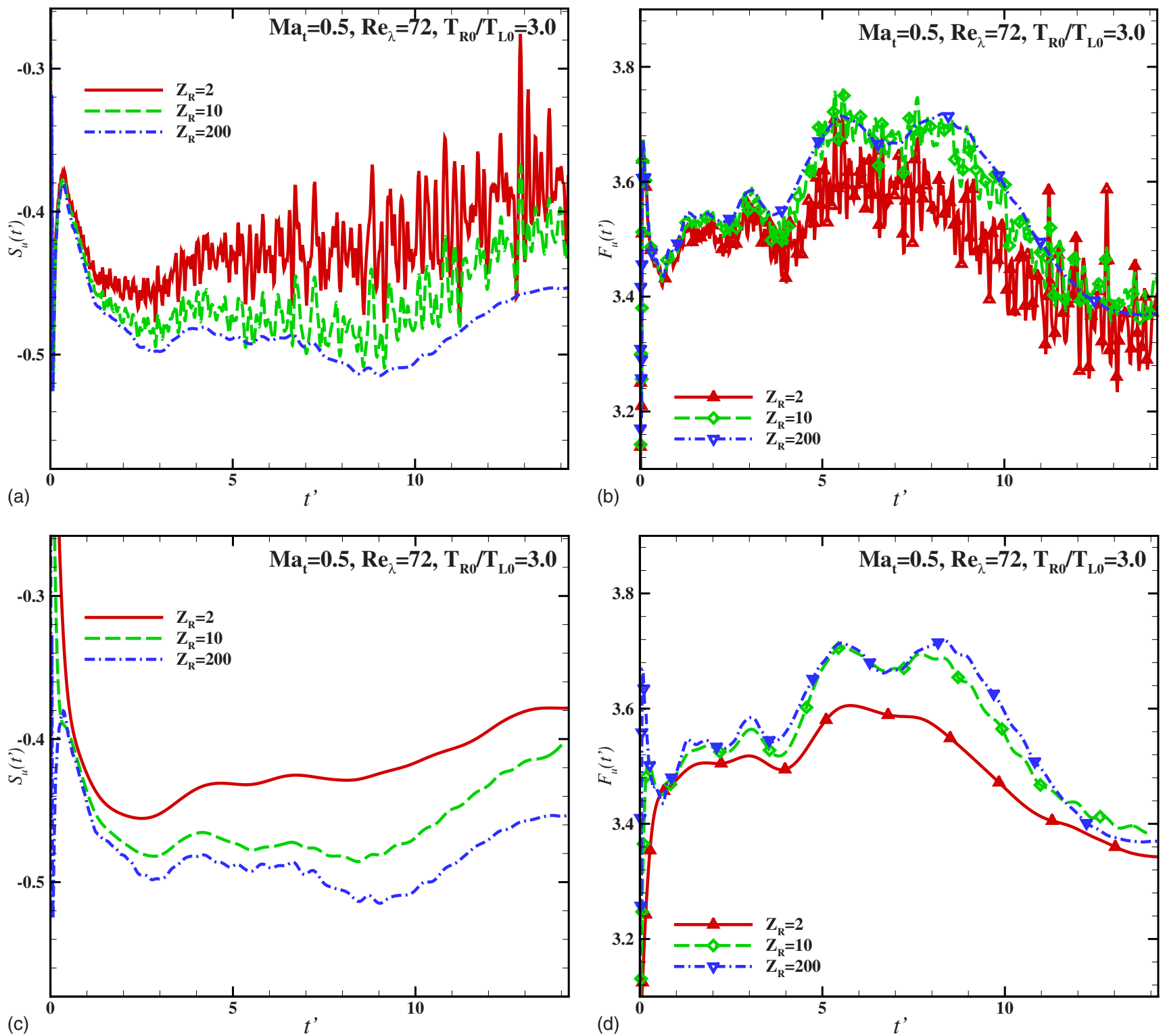

FIG. 4. (Color online) $Z_{R}$ dependence of the skewness $S_{u}$ (left) and the flatness $F_{u}$ (right). $\mathrm{Ma}_{t}=0.5, \mathrm{Re}_{\lambda}=72, N^{3}=128^{3}$. In the bottom row, data are smoothed.

We should also emphasize that the effect of the rotational degrees of freedom on the flow is only through the term $\boldsymbol{\nabla} \cdot\left(\sigma_{R} \boldsymbol{u}\right)=\sigma_{R} \boldsymbol{\nabla} \cdot \boldsymbol{u}+\boldsymbol{u} \cdot \boldsymbol{\nabla} \sigma_{R}$ in Eq. (29d). That is, the rotational energy affects the flows through its direct coupling with the velocity divergence $\boldsymbol{\nabla} \cdot \boldsymbol{u}$. Effectively, the bulk viscosity is no longer a constant in the system. The GKS with the Jeans-Landau-Teller model is ultimately a macroscopic model. A previous study [43] shows that the multitemperature model is necessary for some hypersonic flows. However, its effect on the decaying homogeneous isotropic turbulence is unknown, which will be investigated in this work.

\section{COMPRESSIBLE DECAYING HOMOGENEOUS ISOTROPIC TURBULENCE}

For DHIT, the flow domain is a $3 \mathrm{D}$ cube of size $L^{3}$ $=(2 \pi)^{3}$ with periodic boundary conditions in all three direc- tions. The cube is discretized with a uniform Cartesian mesh size $N^{3}$. The Prandtl number is fixed at $\operatorname{Pr}=0.7$.

A divergence-free random initial velocity field $\boldsymbol{u}_{0}(\boldsymbol{x})$ is generated with a given spectrum by using Rogallo's procedure [52] and a specified root-mean-square (RMS) value

$$
u^{\prime}:=\frac{1}{\sqrt{3}} \sqrt{\langle\boldsymbol{u} \cdot \boldsymbol{u}\rangle} .
$$

We use the following initial energy spectrum $E_{0}(k)$ in the Fourier space $\boldsymbol{k}$ :

$$
E_{0}(k)=A_{0} k^{4} \exp \left(-2 k^{2} / k_{0}^{2}\right),
$$

where the quantities with subscript " 0 " denote the initial values. In what follows, we set $A_{0}=1.3 \times 10^{-4}$ and $k_{0}=8$ unless otherwise stated. 
We will compute the following quantities of turbulence:

$$
\begin{gathered}
K(t):=\frac{1}{2}\langle\rho \boldsymbol{u} \cdot \boldsymbol{u}\rangle, \\
\varepsilon(t):=\left\langle\sum_{i j} \sigma_{i j} \partial_{i} u_{j}\right\rangle, \\
E(k, t)=\int \frac{1}{2} \rho \boldsymbol{u} \cdot \boldsymbol{u} e^{-i k \cdot r} d \boldsymbol{r}, \\
\theta^{\prime}(t):=\sqrt{\left\langle(\nabla \cdot \boldsymbol{u})^{2}\right\rangle}, \\
S_{u}(t)=\frac{1}{3} \sum_{i} S_{u_{i}}, \\
S_{u_{i}}=\frac{\left\langle\left(\partial_{i} u_{i}\right)^{3}\right\rangle}{\left\langle\left(\partial_{i} u_{i}\right)^{2}\right\rangle^{3 / 2}}, \\
F_{u}(t)=\frac{1}{3} \sum_{i} F_{u_{i}}, \\
F_{u_{i}}=\frac{\left\langle\left(\partial_{i} u_{i}\right)^{4}\right\rangle}{\left\langle\left(\partial_{i} u_{i}\right)^{2}\right\rangle^{2}},
\end{gathered}
$$

where $K(t)$ and $\varepsilon(t)$ are the kinetic energy and dissipation rate, respectively, $E(k, t)$ is the energy spectrum, $\theta^{\prime}(t)$ is the RMS of the velocity divergence, $S_{u_{i}}$ and $F_{u_{i}}$ are the skewness and the flatness of the velocity derivative $\partial_{i} u_{i}, i \in\{x, y, z\}$, respectively, and $S_{u}$ and $F_{u}$ are the skewness and the flatness averaged over three directions, respectively. With the initial energy spectrum of Eq. (35), at $t=0$ we have

$$
\begin{gathered}
K_{0}=\frac{3 A_{0}}{64} \sqrt{2 \pi} k_{0}^{5}, \\
\Omega_{0}=\frac{15 A_{0}}{256} \sqrt{2 \pi} k_{0}^{7}, \\
\varepsilon_{0}=2 \frac{\mu_{0}}{\rho_{0}} \Omega_{0},
\end{gathered}
$$

where $K_{0}, \Omega_{0}$, and $\varepsilon_{0}$ are the initial kinetic energy, enthalpy, and dissipation rate, respectively. The dimensional quantities relevant to the DHIT are the Taylor microscale Reynolds number $\mathrm{Re}_{\lambda}$ and the turbulence Mach number $\mathrm{Ma}_{t}$, of which the initial values are

$$
\begin{gathered}
\operatorname{Re}_{\lambda}:=\frac{\langle\rho\rangle u^{\prime} \lambda}{\langle\mu\rangle}=\frac{(2 \pi)^{1 / 4}}{4} \frac{\rho_{0}}{\mu_{0}} \sqrt{2 A_{0}} k_{0}^{3 / 2}, \\
\lambda:=\sqrt{\frac{20 \mu_{0} K_{0}}{\rho_{0} \varepsilon_{0}}},
\end{gathered}
$$

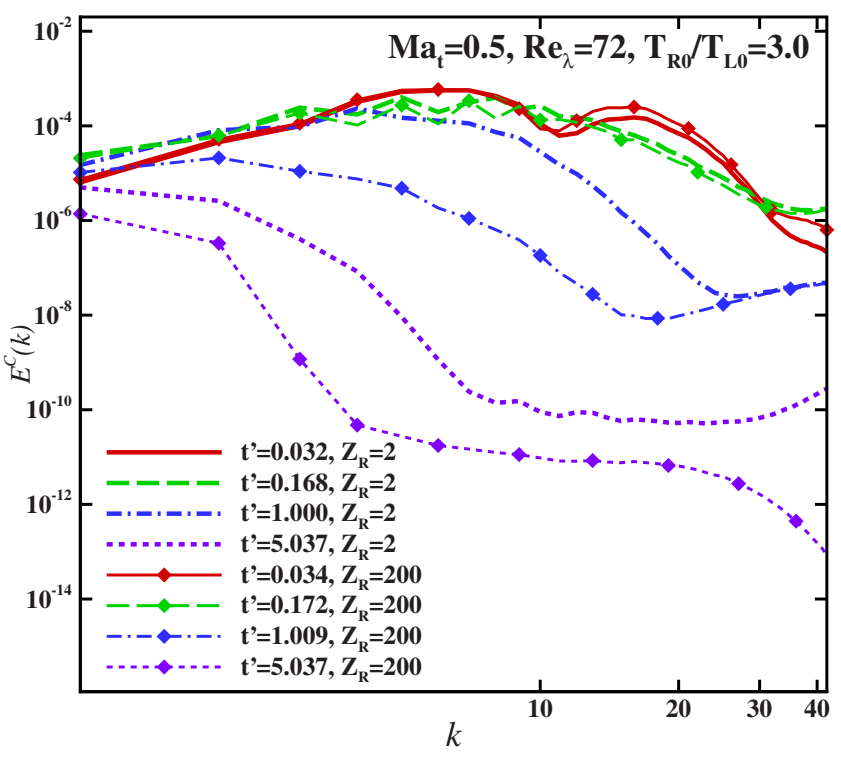

FIG. 5. (Color online) Energy spectrum $E^{C}(k, t)$ of the dilatational component of the velocity with $Z_{R}=2$ and $Z_{R}=200$. $\mathrm{Ma}_{t}=0.5, \operatorname{Re}_{\lambda}=72, N^{3}=128^{3}$.

$$
\mathrm{Ma}_{t}:=\frac{\sqrt{3} u^{\prime}}{\left\langle c_{s}\right\rangle}=\frac{\sqrt{3} u^{\prime}}{\sqrt{\gamma R T_{0}}},
$$

where $\lambda$ is the transverse Taylor microscale length. We set $\rho_{0}=1$ and determine $T_{0}$ and $\mu_{0}$ from the initial values of $\operatorname{Re}_{\lambda}$ and $\mathrm{Ma}_{t}$.

To quantify the compressible effect, we apply the Helmholtz decomposition to the velocity field

$$
\begin{gathered}
\boldsymbol{u}=\boldsymbol{u}^{S}+\boldsymbol{u}^{C}, \\
\boldsymbol{\nabla} \cdot \boldsymbol{u}^{S}=0, \quad \boldsymbol{\nabla} \times \boldsymbol{u}^{C}=0,
\end{gathered}
$$

where $\boldsymbol{u}^{S}$ and $\boldsymbol{u}^{C}$ denote the solenoidal and dilatational components of the velocity field $\boldsymbol{u}$, respectively. The Helmholtz decomposition is unique for homogeneous flows and can be carried out in the Fourier space

$$
\widetilde{\boldsymbol{u}}^{C}=\frac{\boldsymbol{k} \cdot \tilde{\boldsymbol{u}}}{k^{2}} \boldsymbol{k}, \quad \widetilde{\boldsymbol{u}}^{S}=\tilde{\boldsymbol{u}}-\widetilde{\boldsymbol{u}}^{C},
$$

where $\tilde{\boldsymbol{u}}$ denotes the Fourier transform of $\boldsymbol{u}$. Correspondingly, we can decompose both the total kinetic energy $K$ and the dissipation rate $\varepsilon$ into their solenoidal and dilatational components

$$
\begin{gathered}
K=K^{S}+K^{C}, \\
K^{S}=\frac{1}{2}\left\langle\rho \boldsymbol{u}^{S} \cdot \boldsymbol{u}^{S}\right\rangle, \quad K^{C}=\frac{1}{2}\left\langle\rho \boldsymbol{u}^{C} \cdot \boldsymbol{u}^{C}\right\rangle, \\
\varepsilon=\varepsilon^{S}+\varepsilon^{C},
\end{gathered}
$$




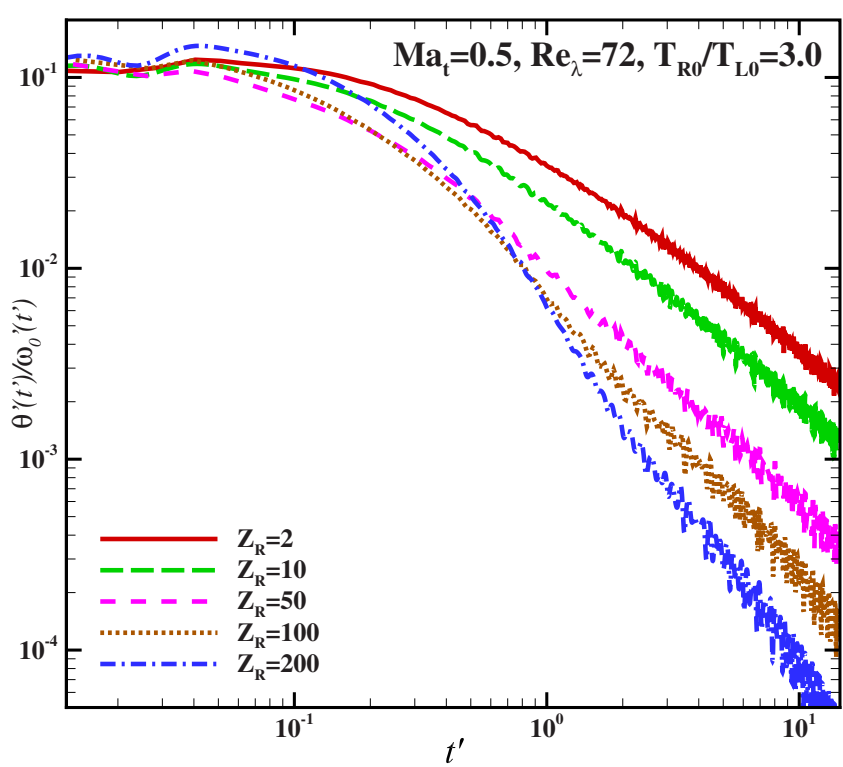

FIG. 6. (Color online) $Z_{R}$ dependence of the RMS velocity divergence $\theta^{\prime} / \omega_{0}^{\prime}$. $\mathrm{Ma}_{t}=0.5, \operatorname{Re}_{\lambda}=72, N^{3}=128^{3}$.

$$
\varepsilon^{S}=\langle\mu \boldsymbol{\omega} \cdot \boldsymbol{\omega}\rangle, \quad \varepsilon^{C}=\left\langle\left(4 \mu / 3+\sigma_{R}\right) \theta^{2}\right\rangle,
$$

where $\boldsymbol{\omega}:=\boldsymbol{\nabla} \times \boldsymbol{u}$ is the vorticity, $\theta:=\boldsymbol{\nabla} \cdot \boldsymbol{u}$ is the dilatation, and $\sigma_{R}$ is given by Eq. (29f). When $T_{R} \approx T_{L}$, Eq. (31) is valid and the formula (39d) for $\varepsilon^{C}$ reduces to

$$
\varepsilon^{C}=\left\langle(4 \mu / 3+\eta) \theta^{2}\right\rangle \text {. }
$$

We will also compute the probability distribution functions (PDFs) for the local Mach number Ma $=\|\boldsymbol{u}\| / c_{s}$ and the shocklet strength $\chi$ defined as follows $[5,22,53]$ :

$$
\begin{gathered}
\chi:=\left(\mathrm{Ma}_{\perp}-1\right), \\
\mathrm{Ma}_{\perp}=\sqrt{1+\left(\frac{\delta u}{2 c_{s}}\right)^{2}}-\frac{\delta u}{2 c_{s}},
\end{gathered}
$$

where $\mathrm{Ma}_{\perp}$ is the normal upstream shock Mach number and $\delta u$ is the two-point longitudinal velocity difference

$$
\delta u(\boldsymbol{r} \mid \delta \boldsymbol{r}):=\delta \hat{\boldsymbol{r}} \cdot[\boldsymbol{u}(\boldsymbol{r})-\boldsymbol{u}(\boldsymbol{r}+\delta \boldsymbol{r})]
$$

and $\delta \hat{\boldsymbol{r}}:=\delta \boldsymbol{r} /\|\delta \boldsymbol{r}\|$ is the unit vector along a chosen direction $r$.

\section{NUMERICAL RESULTS AND DISCUSSIONS}

We will use two resolutions of $N^{3}=128^{3}$ and $256^{3}$ for the flows with the Taylor microscale Reynolds number $\operatorname{Re}_{\lambda}=72$ and 175, respectively. The initial turbulence Mach number is within the range of $0.1 \leq \mathrm{Ma}_{t} \leq 0.5$. Unless otherwise stated,

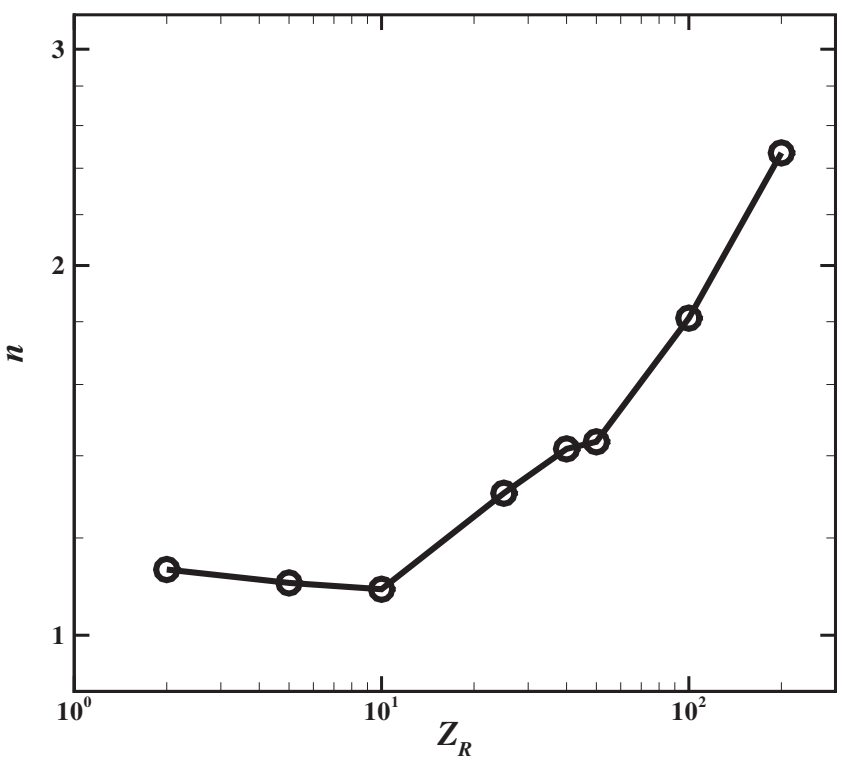

FIG. 7. Dependence of the decay exponent $n$ of $\theta^{\prime}(t) \sim\left(t / t_{0}\right)^{-n}$ on the parameter $Z_{R}$ corresponding to Table I.

we will use the initial turbulence turnover time $t_{0}=K_{0} / \varepsilon_{0}$ to normalize the time, i.e., the dimensionless time $t^{\prime}=t / t_{0}$. We will use the third-order accurate interpolations of Eq. (18) in the flux calculations. Unless otherwise stated, we use the CFL number $\mu_{\mathrm{CFL}}=0.1$. We will investigate the effects of the multitemperature nonequilibrium on the compressible DHIT. Specifically, we will quantify the effects of the parameters $Z_{R}$ and $T_{R 0} / T_{L 0}$ on the statistical quantities relevant to the compressible DHIT, where $T_{R 0}$ and $T_{L 0}$ are the initial values of the rotational and translational temperatures, respectively.

\section{A. Effect of $Z_{R}$}

The Jeans-Landau-Teller model has been widely used to model the energy exchange between the rotational and translational modes (cf., e.g., [12]). The efficacy of the JLT model has been tested with the direct simulation Monte Carlo (DSMC) for an adiabatic relaxation of loaded spheres from a nonequilibrium state [54]. The rotational relaxation time, $Z_{R} \tau$, is strongly temperature dependent [55]. The determination of $Z_{R}$ by theoretical and experimental means is still an active research area [56] which is, however, beyond the scope of the present work. In this work, we will quantify the effect of $Z_{R}$ on the development of the compressible DHIT. We will use $\mathrm{Ma}_{t}=0.5, \mathrm{Re}_{\lambda}=72$, and the ratio of the initial rotational and translational energies $T_{R 0} / T_{L 0}=3.0$, and vary $Z_{R}$ in the range $2 \leq Z_{R} \leq 200$.

Figure 1 shows the evolutions of the rotational and translational temperatures, $T_{R}(t)$ and $T_{L}(t)$ in two time scales, $t / t_{0}$ in the left of Fig. 1 and $t / \tau_{0}$ in the right, where $t_{0}=K_{0} / \varepsilon_{0}$ is

TABLE I. Dependence of the decay exponent $n$ of $\theta^{\prime}(t) \sim\left(t / t_{0}\right)^{-n}$ on the parameter $Z_{R} \cdot \mathrm{Ma}_{t}=0.5$, $T_{R 0} / T_{L 0}=3.0, \operatorname{Re}_{\lambda}=72, N^{3}=128^{3}$.

\begin{tabular}{lccccccc}
\hline \hline$Z_{R}$ & 5 & 10 & 25 & 40 & 50 & 100 & 200 \\
$n$ & 1.103 & 1.090 & 1.305 & 1.418 & 1.437 & 1.812 & 2.470 \\
\hline \hline
\end{tabular}




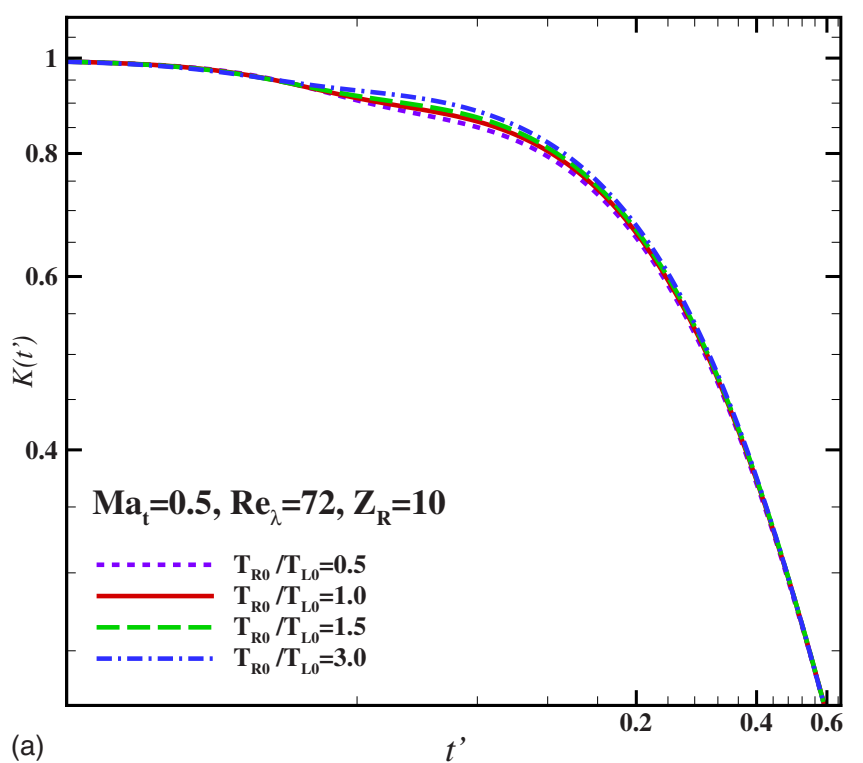

FIG. 8. (Color online) Dependence of the kinetic energy $K(t) / K_{0}$ (left) and the dissipation rate $\varepsilon(t) / \varepsilon_{0}\left(\right.$ right) on $T_{R 0} / T_{L 0}$. Ma $t_{t}=0.5$, $\operatorname{Re}_{\lambda}=72, N^{3}=128^{3}$.

the initial turbulence turnover time, $\tau_{0}$ is the initial relaxation time, and $t_{0} \gg \tau_{0}$. Both temperatures, $T_{L}$ and $T_{R}$, evolve monotonically to their common equilibrium value. Clearly, the larger the $Z_{R}$ is, the longer it takes for $T_{L}$ and $T_{R}$ to equilibrate. We note that the time for the temperatures to reach their equilibrium is relatively short in terms of the initial turbulence turnover time $t_{0}$; even with $Z_{R}=200$, the equilibration is completed at about $t / t_{0} \approx 0.1$. This means that the temperature relaxation time is usually shorter than a typical hydrodynamic time scale. For $Z_{R}=2$, the temperatures equilibrate rather quickly; the equilibration is completed at $t / \tau_{0} \approx 5$.

Although the relaxation process of rotational temperature is completed rather quickly during the initial stage of the decaying turbulence, it still exerts an influence on the decay-

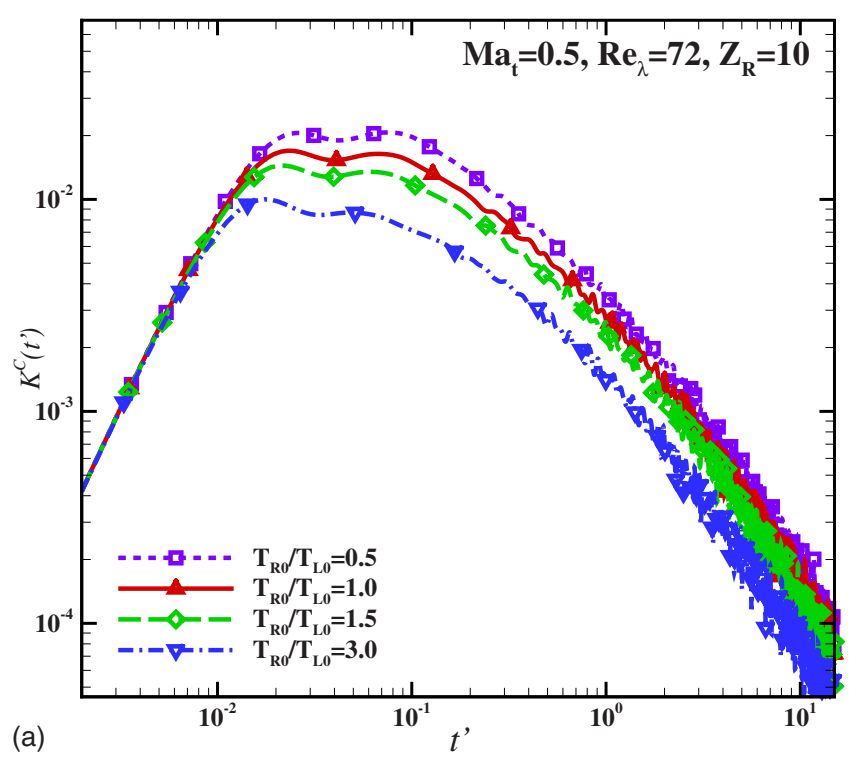

ing process of isotropic turbulence. Figure 2 shows the evolution of the kinetic energy $K(t) / K_{0}$ and the dissipation rate $\varepsilon(t) / \varepsilon_{0}$ for different $Z_{R}$. The effect of $Z_{R}$ on $K(t) / K_{0}$ and $\varepsilon(t) / \varepsilon_{0}$ is invisible and similarly for their solenoidal components, $K^{S}(t) / K_{0}$ and $\varepsilon^{S}(t) / \varepsilon_{0}$, as shown in Fig. 3. However, $Z_{R}$ has a considerable effect on the dilatational components of the energy and the dissipation rate, $K^{C}(t) / K_{0}$ and $\varepsilon^{C}(t) / \varepsilon_{0}$, also shown in Fig. 3. The larger the $Z_{R}$, the smaller the $K^{C}(t) / K_{0}$ and $\varepsilon^{C}(t) / \varepsilon_{0}$ after a short initial period of time $t / t_{0} \approx 0.1$. Because $\varepsilon_{0}$ is computed by using Eq. (40), which does not account for the nonequilibrium effect when $T_{L}$ and $T_{R}$ are far apart at the initial stage, $\varepsilon(t) / \varepsilon_{0}$ is not close to 1 initially in Fig. 3 for some cases.

In Fig. 4, we show the evolution of the skewness $S_{u}(t)$ and the flatness $F_{u}(t)$ of the velocity derivative $\partial_{i} u_{i}$ in the decay-

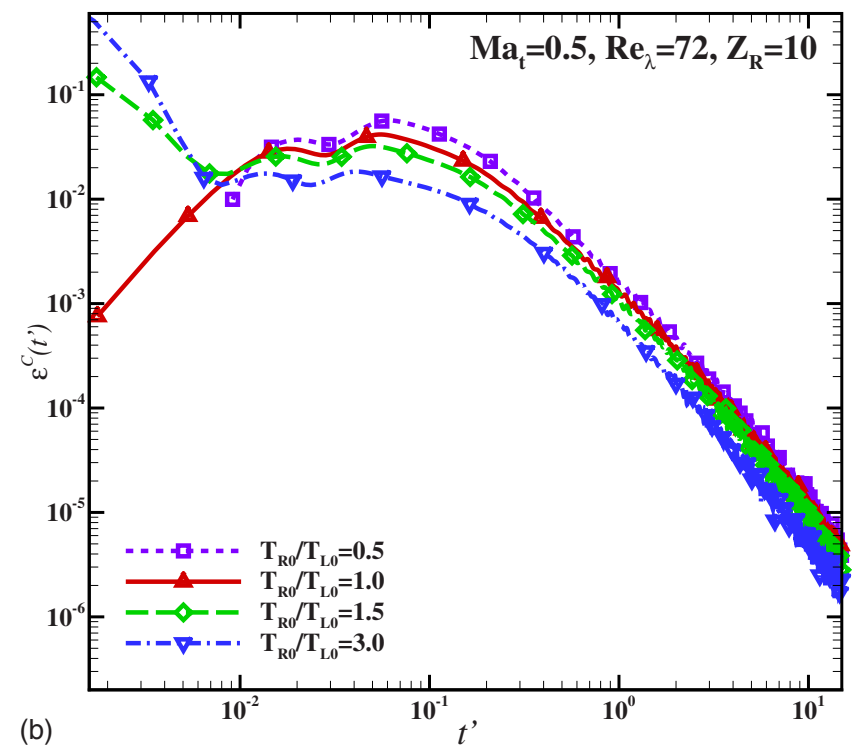

FIG. 9. (Color online) Dependence of the dilatational components of the kinetic energy $K^{C}(t) / K_{0}$ (left) and the dissipation rate $\varepsilon^{C}(t) / \varepsilon_{0}$ (right) on $T_{R 0} / T_{L 0} \cdot \mathrm{Ma}_{t}=0.5, \mathrm{Re}_{\lambda}=72, N^{3}=128^{3}$. 

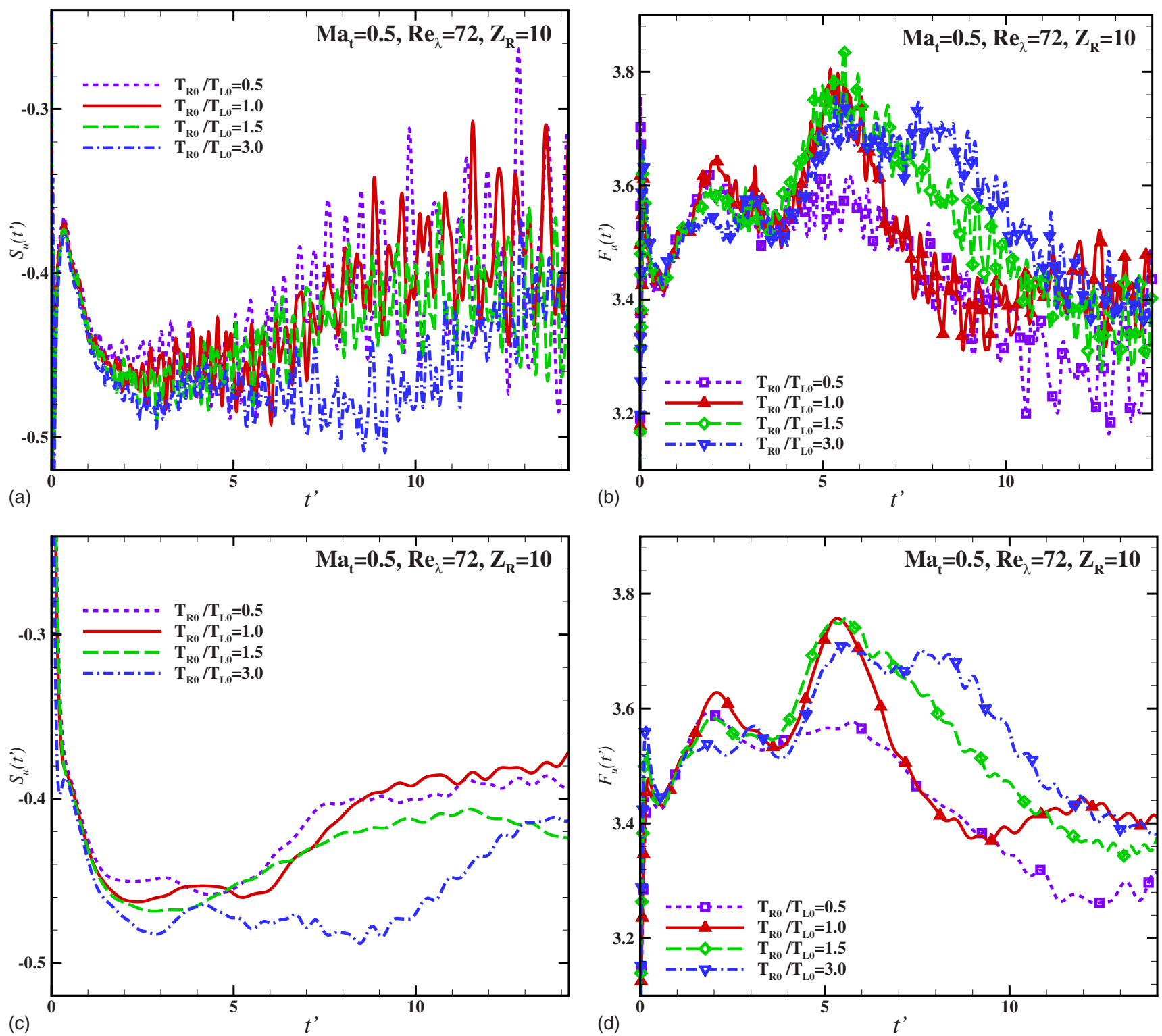

FIG. 10. (Color online) Dependence of the skewness $S_{u}$ (left), $F_{u}$ (right) on the ratio $T_{R 0} / T_{L 0}$. $\mathrm{Ma}_{t}=0.5, \operatorname{Re}_{\lambda}=72, N^{3}=128^{3}$. In the bottom row, data are smoothed.

ing process. The skewness involves the second- and fourthorder derivatives of the velocity field due to the following formula [57]:

$$
S_{u}(t)=\frac{3 \sqrt{30}}{14} \frac{\left[2 \int_{0}^{\infty} k^{4} E(k, t) d k+\frac{d}{d t} \int_{0}^{\infty} k^{2} E(k, t) d k\right]}{\left[\int_{0}^{\infty} k^{2} E(k, t) d k\right]^{3 / 2}},
$$

where $E(k, t)$ is the energy spectrum. Therefore, it is sensitive to the variations in the velocity field. Two observations can be made. First, the magnitude of $S_{u}(t)$ is larger and closer to -0.5 as $Z_{R}$ increases. And second, the magnitude of oscillations in $S_{u}$ decreases as $Z_{R}$ increases. These observations are consistent with the correlation between the compressible (dilatational) component of the flow and $Z_{R}$. Based on Eq. (7), the larger the $Z_{R}$ is, the weaker is the source term, which is proportional to $\left(T_{R}-T_{R}^{*}\right)$, hence the weaker the effect due to multitemperature relaxation, which mimics the internal degrees of freedom in diatomic gases. In the limit of $Z_{R} \rightarrow \infty$, both the source term $s$ in Eq. (7) and $\sigma_{R}$ in Eq. (29f) vanish and the macroscopic equations (29) derived from the multitemperature model reduce to the usual compressible Navier-Stokes equations (31).

To further demonstrate the effect of $Z_{R}$, we show in Fig. 5 the energy spectrum $E^{C}(k, t)$ for the dilatational component of the velocity with $Z_{R}=2$ and 200 . The energy spectrum $E^{C}(k, t)$ is shown in four times: $t_{1}^{\prime} \approx 0.03$ in the very early stage of the decay, $t_{2}^{\prime} \approx 0.17$ when $\varepsilon\left(t^{\prime}\right) / \varepsilon_{0}$ reaches its peak, $t_{3}^{\prime} \approx 1.0$ when $K\left(t^{\prime}\right) / K_{0}$ decays about 1 order of magnitude, and $t_{4}^{\prime} \approx 5.0$ when $K\left(t^{\prime}\right) / K_{0}$ decays about 2 orders of magnitude. We note that the variation of $Z_{R}$ has little effect on 
$E^{C}(k, t)$ in the initial stage of the decay. However, in the late stage of the decay, when viscous effect is dominant, the effect due to the variation of $Z_{R}$ becomes ostensibly noticeable. This is because $Z_{R}$ directly affects the dissipation of $e_{R}$. The larger $Z_{R}$, the longer it takes to equilibrate $T_{R}$, hence a larger fraction of energy is dissipated after the equilibration.

In Fig. 6, we show the $Z_{R}$ dependence of the normalized RMS velocity divergence $\theta^{\prime} / \omega_{0}^{\prime}$, where $\omega_{0}^{\prime}$ is the initial averaged vorticity. Clearly, the normalized RMS velocity divergence strongly depends on $Z_{R}$. After an initial period of time, $\theta^{\prime} / \omega_{0}^{\prime}$ decays significantly faster when $Z_{R}$ is sufficiently large. The compressibility is ostensibly stronger in the stage of final decay [58] when $Z_{R}$ is smaller. This is also corroborated by both the skewness $S_{u}(t)$ and the flatness $F_{u}(t)$ shown in Fig. 4. When $Z_{R}=200$, in the time interval of $2<t^{\prime}<11$, $S_{u}$ stays very close to -0.5 , which is the theoretical value for the incompressible decaying turbulence. In contrast, when $Z_{R}=2$ and in the same time interval, $S_{u}$ is about -0.42 . The magnitude of oscillations in both $S_{u}(t)$ and $F_{u}(t)$ increases as $Z_{R}$ decreases. This effect is similar to that of increasing the Mach number, as observed previously [24]. This is because the compressibility in the flow is relatively stronger with smaller $Z_{R}$ and so is the strength of shocklets in the system. Consequently, the magnitudes of local gradients are larger, leading to larger oscillations in both $S_{u}(t)$ and $F_{u}(t)$, both of which depend on velocity derivatives.

It is also interesting to note that the effect of $Z_{R}$ is much more noticeable in the late stage of the flow, i.e., after the dissipation rate $\varepsilon(t)$ reaches its peak (at about $t^{\prime} \approx 0.17$, cf. the right figure in Fig. 2). As shown in Fig. 6, the decay rate of $\theta^{\prime}(t)$ depends on $Z_{R}$. Assuming that in the late stage of the flow $\theta^{\prime}\left(t^{\prime}\right) / \omega_{0}^{\prime}$ decays as

$$
\frac{\theta^{\prime}\left(t^{\prime}\right)}{\omega_{0}^{\prime}} \sim\left(t / t_{0}\right)^{-n},
$$

we compute the decay exponent $n$ depending on $Z_{R}$ and the results are summarized in Table I and Fig. 7. There seems to be a transition in $n$ taking place at some point $Z_{R}>10$ : the exponent $n$ varies very little when $Z_{R} \leq 10$, while the exponent $n$ grows linearly as $Z_{R}$ increases when $Z_{R} \geq 10$. The parameter $Z_{R}$ only affects the dilatation and because the dilatational components of the kinetic energy and the dissipation rate are relatively small (about $1 \%$ for $\mathrm{Ma}_{t}=0.5$ ), the effects of $Z_{R}$ on the kinetic energy and the dissipation rate are hardly observable, although they can be clearly seen in the skewness $S_{u}(t)$ and the flatness $F_{u}(t)$. The effect of the $Z_{R}$ will be further discussed in Sec. IV C.

\section{B. Effect of the initial state $T_{R 0} / T_{L 0}$}

The initial ratio of rotational and translational temperatures $R_{T}:=T_{R 0} / T_{L 0}$ can affect the decay turbulence. In this section, we will quantify the effect due to the ratio $T_{R 0} / T_{L 0}$ with various initial conditions. In what follows, we will use $Z_{R}=10$, which is consistent with the values used by others [12,54].

We will first compute the compressible DHIT with $\mathrm{Ma}_{t}$ $=0.5, \mathrm{Re}_{\lambda}=72, \mathrm{Zr}=10$, and various values of $T_{R 0} / T_{L 0}$. In Fig. 8, we show the dependence of the total kinetic energy

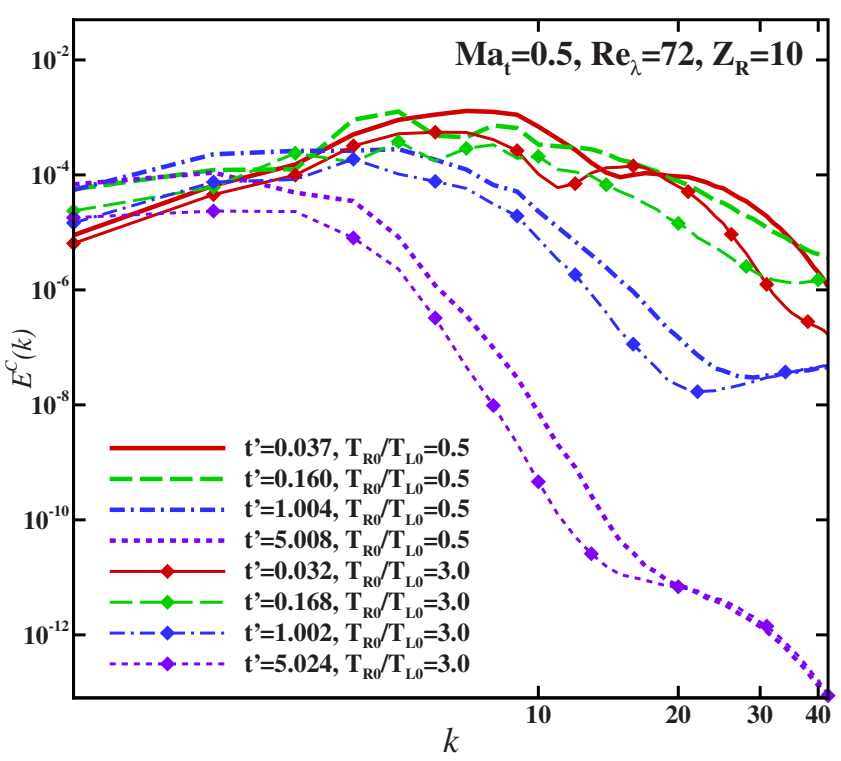

FIG. 11. (Color online) Energy spectrum $E^{C}(k, t)$ of the dilatational component of the velocity with $T_{R 0} / T_{L 0}=0.5$ and $T_{R 0} / T_{L 0}$ $=3 . \mathrm{Ma}_{t}=0.5, \operatorname{Re}_{\lambda}=72, N^{3}=128^{3}$.

$K(t) / K_{0}$ and the dissipation rate $\varepsilon(t) / \varepsilon_{0}$ on the ratio $T_{R 0} / T_{L 0}$. Clearly, the effects of $T_{R 0} / T_{L 0}$ on the total kinetic energy $K(t) / K_{0}$ and the dissipation rate $\varepsilon(t) / \varepsilon_{0}$ are more visible than that of $Z_{R}$, as shown in Fig. 2. As the ratio $T_{R 0} / T_{L 0}$ increases, so do $K(t) / K_{0}$ and $\varepsilon(t) / \varepsilon_{0}$, but they increase only a few percent when $0.5 \leq T_{R 0} / T_{L 0} \leq 3.0$. The effects of $T_{R 0} / T_{L 0}$ are much more prominent on the compressible components of the kinetic energy and the dissipation rate, $K^{C}(t) / K_{0}$ and $\varepsilon^{C}(t) / \varepsilon_{0}$, as shown in Fig. 9. As the ratio $T_{R 0} / T_{L 0}$ increases, both $K^{C}(t) / K_{0}$ and $\varepsilon^{C}(t) / \varepsilon_{0}$ decrease. This is because a larger $T_{R 0} / T_{L 0}$ leads to a higher equilibrium (translational) temperature $T$ after the rotational temperature relaxes. Consequently, the speed of sound $c_{s}$ increases overall and, in turn, the Mach number decreases; hence the compressibility is reduced and vice versa. When $T_{R}=T_{L}$, there is no equilibration process between the two temperatures. Thus, $T_{R 0}<T_{L 0}$ and $T_{R 0}>T_{L 0}$ correspond to two scenarios: compression and expansion, respectively.

The effect of the ratio $T_{R 0} / T_{L 0}$ on the skewness $S_{u}(t)$ and the flatness $F_{u}(t)$ is shown in Fig. 10. A smaller $T_{R 0} / T_{L 0}$ leads a stronger compressible effect, which in turn strengthens the shocklets and the gradients in the flow field. In Fig. 10, we can clearly observe that, after the initial equilibration, the smaller is the ratio $T_{R 0} / T_{L 0}$, the smaller the magnitude of $S_{u}(t)$, the more oscillatory $S_{u}(t)$, and the more $S_{u}(t)$ deviates away from the value of -0.5 . As for the flatness $F_{u}(t)$, the smaller is the ratio $T_{R 0} / T_{L 0}$, the larger is the $F_{u}(t)$. By comparing Fig. 10 to Fig. 4, we can see that the effects of the ratio $T_{R 0} / T_{L 0}$ on the skewness $S_{u}(t)$ and the flatness $F_{u}(t)$ are very similar to that of $Z_{R}$.

In Fig. 11, we show the energy spectrum $E^{C}(k, t)$ for the dilatational component of the velocity with $T_{R 0} / T_{L 0}=0.5$ and 3. In contrast to Fig. 5 for the effect of $Z_{R}$ on $E^{C}(k, t)$, we can see that the effect of $T_{R 0} / T_{L 0}$ is clearly observable from the beginning of the decay process. The larger the $T_{R 0} / T_{L 0}$ is, the weaker the $E^{C}(k, t)$. This is because a larger $T_{R 0} / T_{L 0}$ leads to 

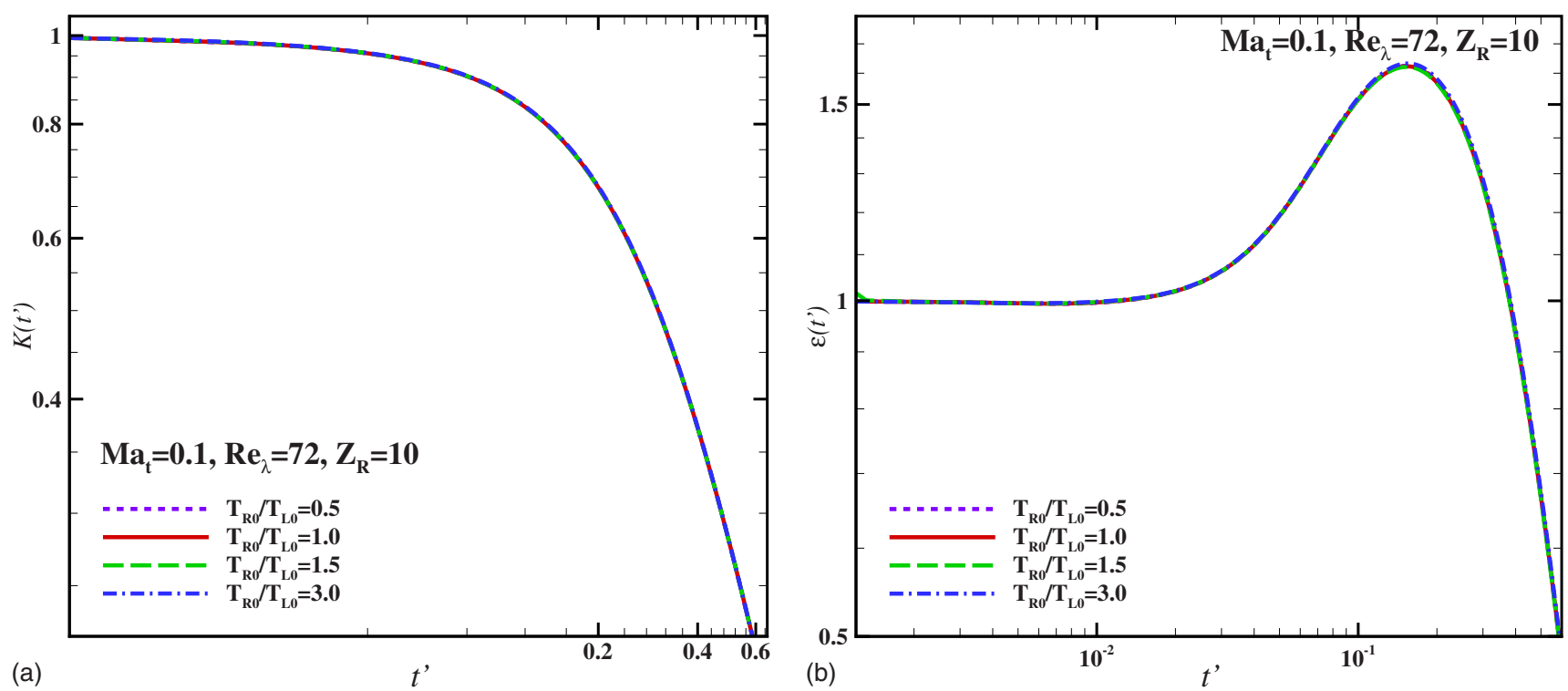

FIG. 12. (Color online) Dependence of the kinetic energy $K(t) / K_{0}$ (left) and dissipation rate $\varepsilon(t) / \varepsilon_{0}$ (right) on the ratio $T_{R 0} / T_{L 0}$. $\mathrm{Ma}_{t}=0.1, \operatorname{Re}_{\lambda}=72, N^{3}=128^{3}$.

higher equilibrium (translational) temperature $T$ and in turn, lower Mach numbers over the entire system after rotational temperature relaxes quickly.

\section{Effect of $\mathrm{Ma}_{t}$ and $\mathrm{Re}_{\boldsymbol{\lambda}}$}

We now investigate the effects of the initial turbulence Mach number $\mathrm{Ma}_{t}$ and the Taylor microscale Reynolds number $\operatorname{Re}_{\lambda}$. We first show results with a lower turbulence Mach number $\mathrm{Ma}_{t}=0.1$. In Fig. 12, we show the kinetic energy $K(t) / K_{0}$ and the dissipation rate $\varepsilon(t) / \varepsilon_{0}$ with $\mathrm{Ma}_{t}=0.1$ and varying $T_{R 0} / T_{L 0}$. The effects of $T_{R 0} / T_{L 0}$ are hardly visible in $K(t) / K_{0}$ and $\varepsilon(t) / \varepsilon_{0}$; however, they are clearly seen in the compressible components of the kinetic energy, $K^{C}(t) / K_{0}$,

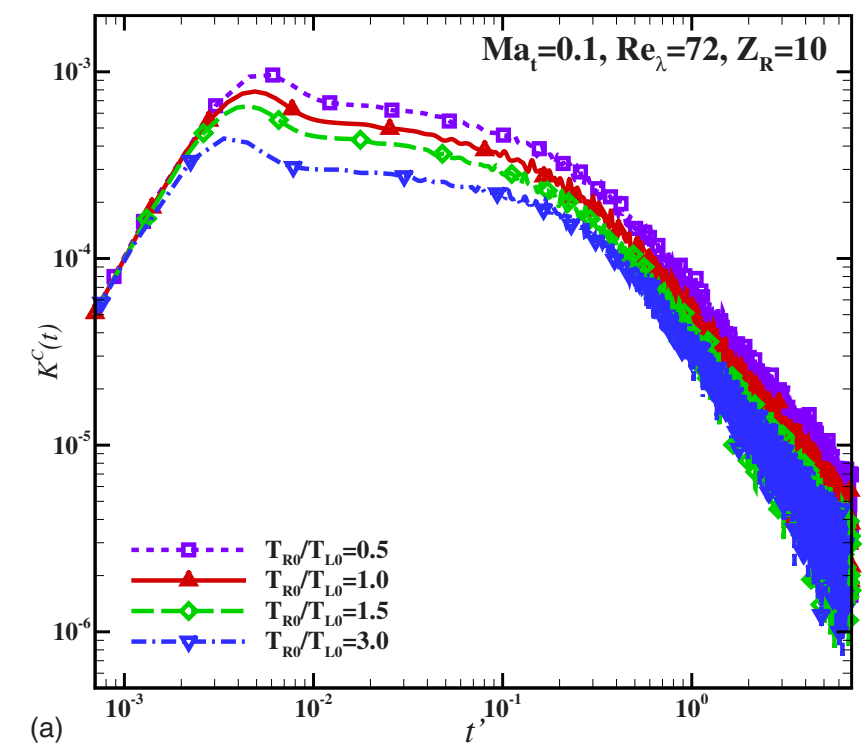

and the dissipation rate, $\varepsilon^{C}(t) / \varepsilon_{0}$, as shown in Fig. 13. We note that both $K^{C}(t) / K_{0}$ and $\varepsilon^{C}(t) / \varepsilon_{0}$ are reduced more than 1 order of magnitude when $\mathrm{Ma}_{t}$ decreases from 0.5 to 0.1 , as clearly seen in Figs. 13 and 9. Also, the dependence of $K^{C}(t) / K_{0}$ and $\varepsilon^{C}(t) / \varepsilon_{0}$ on $T_{R 0} / T_{L 0}$ is weaker at lower Mach number.

We can clearly see the effect of the turbulence Mach number $\mathrm{Ma}_{t}$ on the flow by comparing Fig. 13 to Fig. 9. To further demonstrate the Mach-number effect, we show the normalized RMS velocity divergence $\theta^{\prime}(t) / \omega_{0}^{\prime}$ in Fig. 14 and compare to its counterpart with $\mathrm{Ma}_{t}=0.5$ in Fig. 6 . Since the initial velocity $\boldsymbol{u}_{0}$ is divergent free, i.e., $\boldsymbol{\nabla} \cdot \boldsymbol{u}=0$, the normalized RMS velocity divergence $\theta^{\prime}(t) / \omega_{0}^{\prime}$ grows monotonically after a very short initial period of time to reach its first maxi-

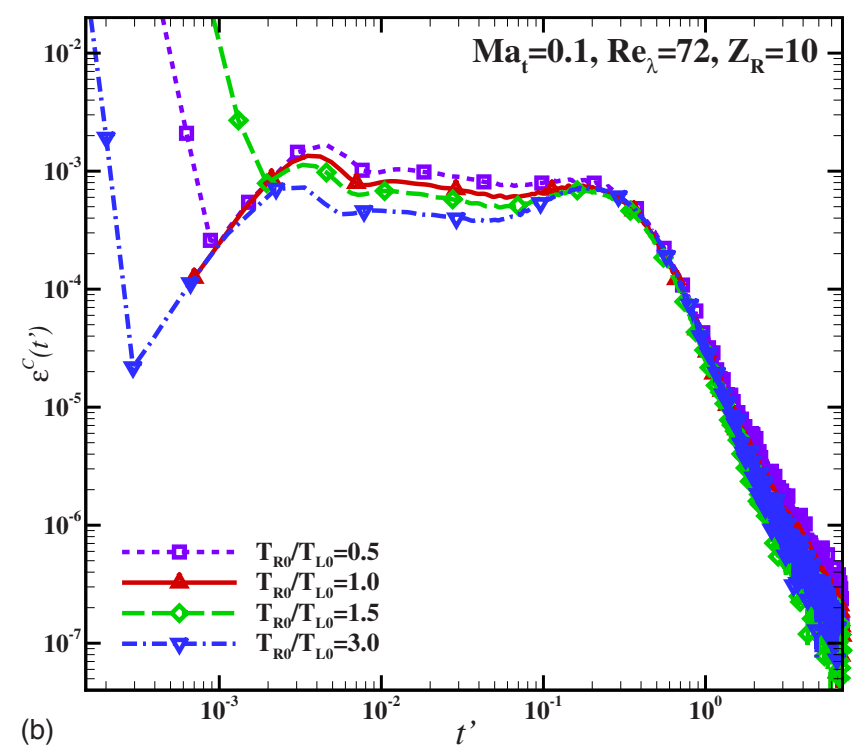

FIG. 13. (Color online) Evolution of the dilatational component of the kinetic energy, $K^{C}(t) / K_{0}$, (left) and the dissipation rate, $\varepsilon^{C}(t) / \varepsilon_{0}$, (right) for different $T_{R 0} / T_{L 0}$ with $\mathrm{Ma}_{t}=0.1, \mathrm{Re}_{\lambda}=72$, and $N^{3}=128^{3}$. 


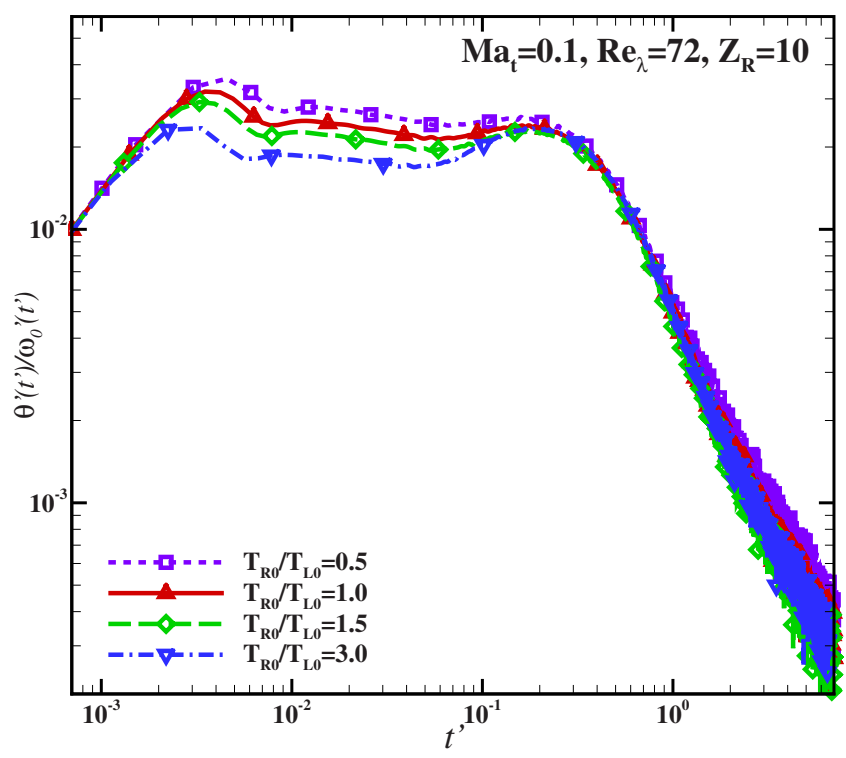

FIG. 14. (Color online) $T_{R 0} / T_{L 0}$ dependence of the RMS velocity divergence $\theta^{\prime} / \omega_{0}^{\prime}$. $\mathrm{Ma}_{t}=0.1, \operatorname{Re}_{\lambda}=72, N^{3}=128^{3}$.

mum, as shown in Fig. 14; it levels off a little before reaching its second maximum and then enters the final decaying process [58]. The second peak of $\theta^{\prime}(t) / \omega_{0}^{\prime}$ coincides with that of $\varepsilon(t) / \varepsilon_{0}$, indicating that the second peak is due to the nonlinearity of the flow. With a larger turbulence Mach number, e.g., $\mathrm{Ma}_{t}=0.5$, the production of $\theta^{\prime}$ will last longer, while the time when the dissipation rate $\varepsilon(t)$ reaches its peak changes relatively little, hence two peaks $\theta^{\prime}(t) / \omega_{0}^{\prime}$ almost merge together in the case of $\mathrm{Ma}_{t}=0.5$, as shown in Fig. 6. The behavior of $\varepsilon^{C}(t) / \varepsilon_{0}$ is very much similar to that of $\theta^{\prime}(t) / \omega_{0}^{\prime}$ because the term of $4\left\langle\mu \theta^{2}\right\rangle / 3$ predominates in $\varepsilon^{C}(t)$. The behavior of $K^{C}(t) / K_{0}$ is also similar to that of $\theta^{\prime}(t) / \omega_{0}^{\prime}$, except that $K^{C}(t) / K_{0}$ does not have the second maximum.

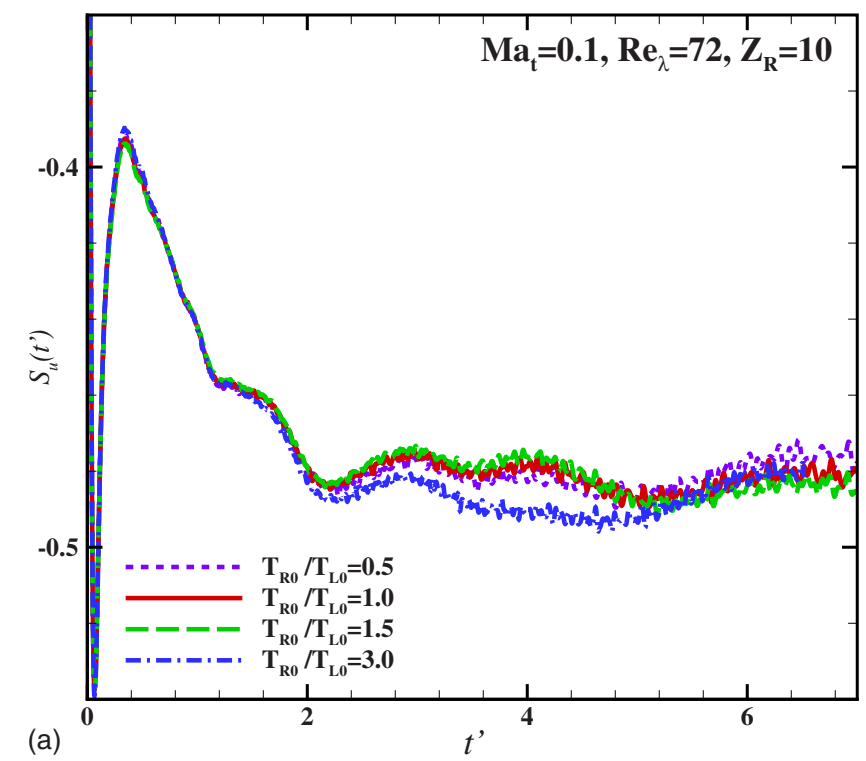

In Fig. 15, we show the dependence of the skewness $S_{u}(t)$ and the flatness $F_{u}(t)$ on the ratio $T_{R 0} / T_{L 0}$ with $\mathrm{Ma}_{t}=0.1$. Clearly, the dependence of both $S_{u}(t)$ and $F_{u}(t)$ on $T_{R 0} / T_{L 0}$ is much weaker than that with $\mathrm{Ma}_{t}=0.5$. The variations of both $S_{u}(t)$ and $F_{u}(t)$ are rather insignificant in this case.

We next investigate the effect due to the initial Taylor Reynolds number $\operatorname{Re}_{\lambda}$. We use a higher initial Taylor Reynolds number $\operatorname{Re}_{\lambda}=175, \mathrm{Ma}_{t}=0.5$, and $N^{3}=256^{3}$, with varying $T_{R 0} / T_{L 0}$. In Fig. 16, we show the kinetic energy $K(t) / K_{0}$ and the dissipation rate $\varepsilon(t) / \varepsilon_{0}$. Similar to the previous cases, the effect of $T_{R 0} / T_{L 0}$ on both $K(t) / K_{0}$ and $\varepsilon(t) / \varepsilon_{0}$ is very weak. The larger Reynolds number does increase the peak value of the dissipation rate $\varepsilon(t) / \varepsilon_{0}$ because of stronger nonlinearity.

In Fig. 17, we show the dependence of the compressible components of the energy, $K^{C}(t) / K_{0}$, and the dissipation rate, $\varepsilon^{C}(t) / \varepsilon_{0}$, on the ratio $T_{R 0} / T_{L 0}$. Comparing Fig. 17 to Fig. 9, it is clear that the Reynolds number has little effect on the compressible components of the kinetic energy $K^{C}(t) / K_{0}$ and the dissipation rate $\varepsilon^{C}(t) / \varepsilon_{0}$. In Fig. 18, we show the dependence of the skewness $S_{u}(t)$ and the flatness $F_{u}(t)$ on the ratio $T_{R 0} / T_{L 0}$. Again, the Reynolds number does not have a significant effect on the overall feature of $S_{u}(t)$ and $F_{u}(t)$. However, the magnitude of oscillations in both $S_{u}(t)$ and $F_{u}(t)$ does increase significantly, especially in the case of $T_{R 0} / T_{L 0}=3.0$.

The above results show that the turbulence Mach number $\mathrm{Ma}_{t}$ is the dictating factor which determines the compressibility in the compressible DHIT, as expected. The multitemperature model significantly enhances the versatility of the compressible Navier-Stokes equations to simulate the nonequilibrium flows. In Fig. 19, we show the dependence of the normalized RMS velocity divergence $\theta^{\prime}(t) / \omega_{0}$ on the parameter $Z$, which adjusts the bulk viscosity $\eta$ through Eq. (33) and the initial turbulence Mach number $\mathrm{Ma}_{t}$. In the former case, the initial turbulence Mach number $\mathrm{Ma}_{t}=0.5$ and in the latter case, the parameter $Z=2$.

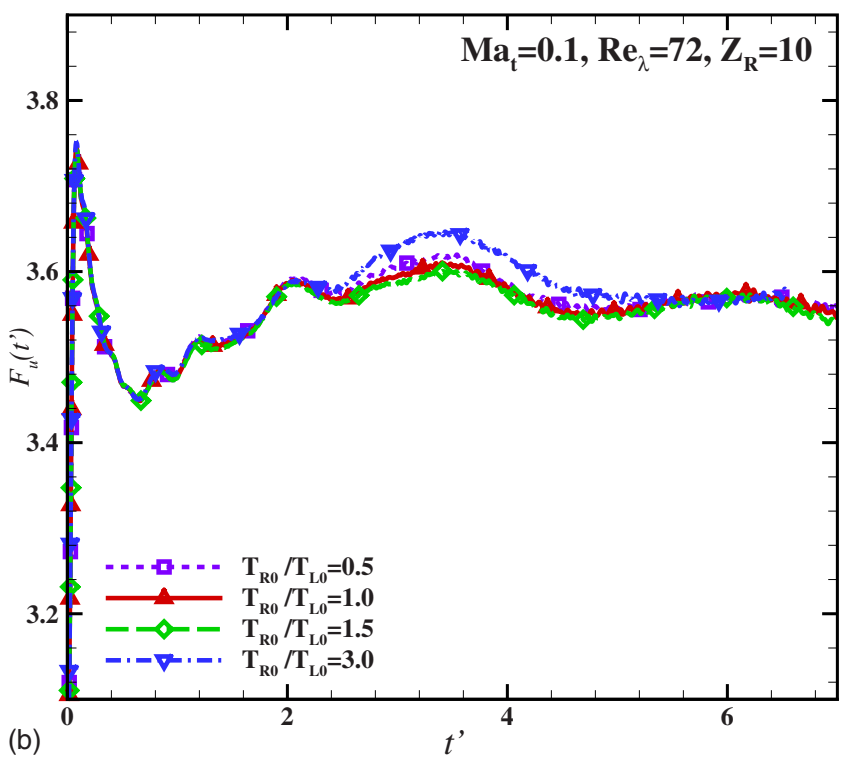

FIG. 15. (Color online) Dependence of the skewness $S_{u}$ (left) and the flatness $F_{u}(t)$ (right) on the ratio $T_{R 0} / T_{L 0} \cdot \mathrm{Ma}_{t}=0.1, \mathrm{Re}_{\lambda}=72$, $N^{3}=128^{3}$. 

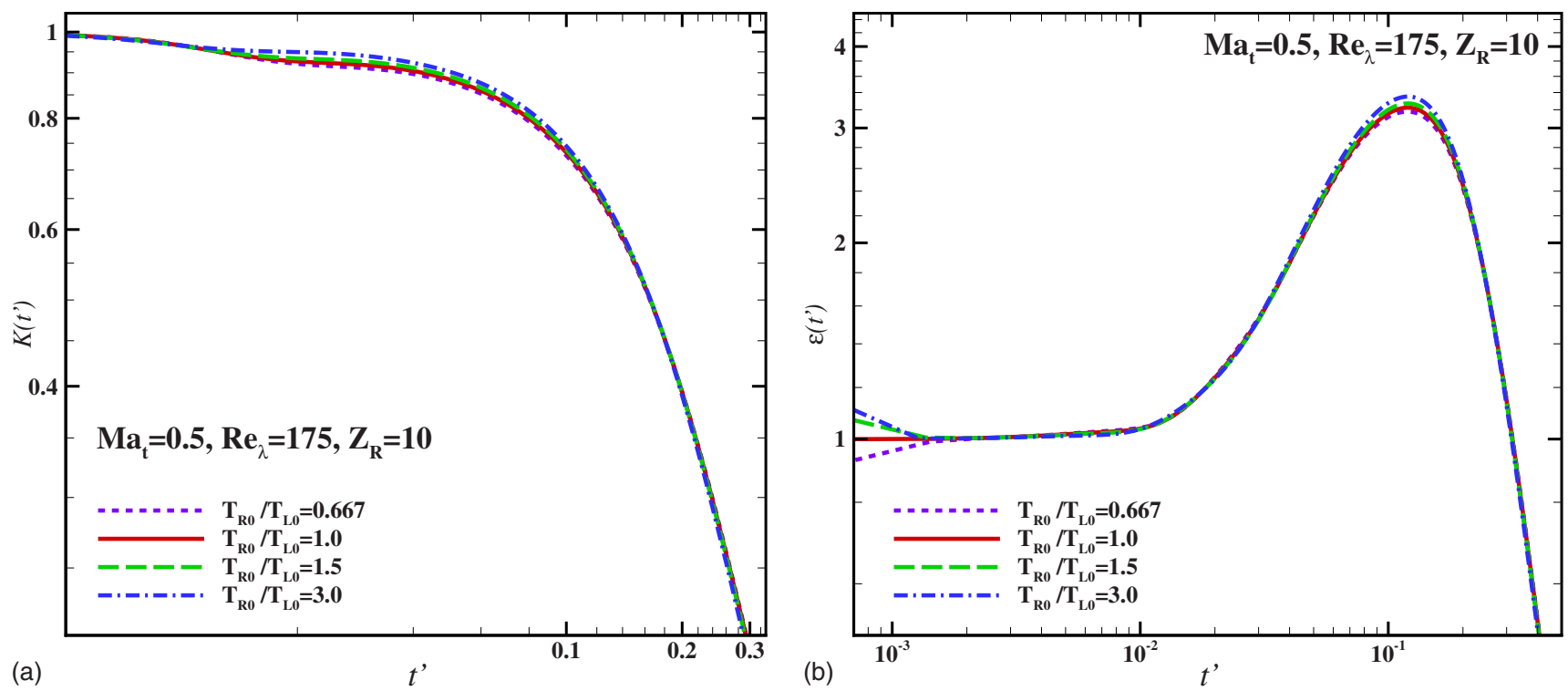

FIG. 16. (Color online) Dependence of the kinetic energy $K(t) / K_{0}$ (left) and dissipation rate $\varepsilon(t) / \varepsilon_{0}$ (right) on the ratio of $T_{R 0} / T_{L 0}$. $\mathrm{Ma}_{t}=0.5, \operatorname{Re}_{\lambda}=175, N^{3}=256^{3}$.

A comparison of Fig. 19 (left) and Fig. 6 shows that the effects of $Z$ (or the bulk viscosity $\eta$ ) and $Z_{R}$ are similar in the sense that they affect the decay of $\theta^{\prime}(t)$ in its late stage. However, the effect of $Z_{R}$ is far more prominent than that of $Z$ because the multitemperature model includes the dynamics of an additional internal energy beyond the translational temperature, which significantly affects the dynamics of the system. We compute the decay exponent $n$ for $\theta^{\prime}\left(t^{\prime}\right)$ from the data in Fig. 19 (left): $n=1.174,1.170$, and 1.151, corresponding to $Z=0,2$, and 5, respectively. As $Z$ increases, also does the bulk viscosity $\eta$. The result indicates that increase of the bulk viscosity leads to slower decay of the dilatational terms in the flow.

A comparison of Fig. 19 (right) and Fig. 14 clearly shows the similarity between the effect due to the initial ratio

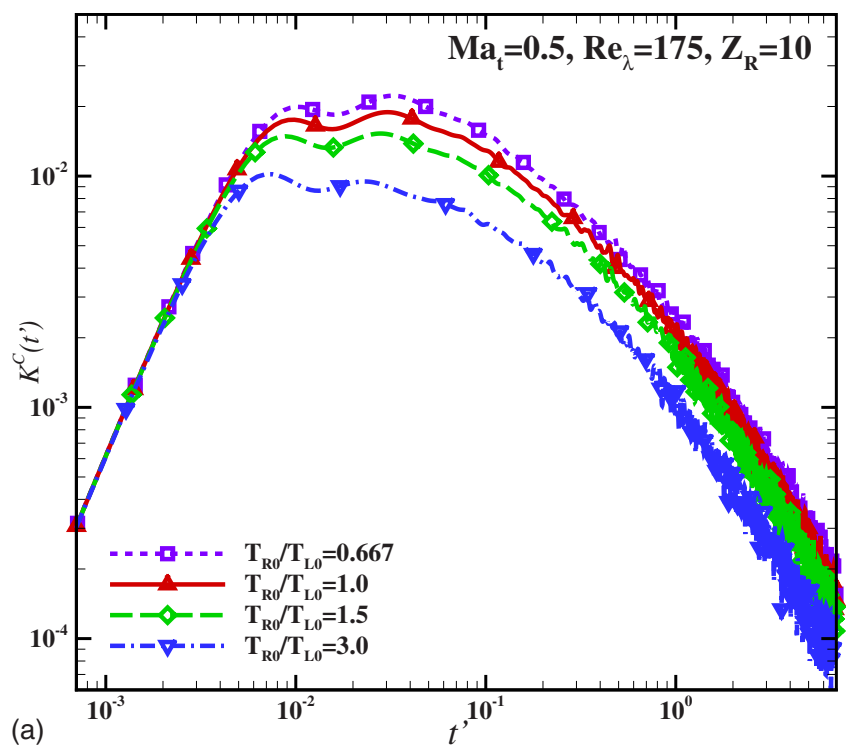

$T_{R 0} / T_{L 0}$ and the initial turbulence Mach number $\mathrm{Ma}_{t}$. The ratio $T_{R 0} / T_{L 0}$ specifies the initial state of the flow, i.e., how much energy is given to the internal degrees of freedom initially. The initial state directly affects the Mach number in the system: when $T_{R 0} / T_{L 0}>1$, the equilibrium temperature $T$ is raised, thus the averaged Mach number is reduced; similarly, when $T_{R 0} / T_{L 0}<1$, the equilibrium temperature is lowered, hence the averaged Mach number is enhanced.

\section{PDFs of the local Mach number Ma and the shock strength $\chi$}

In this section, we will study the probability distribution functions of the local Mach number $\mathrm{Ma}$ and the shock strength $\chi$. Figure 20 presents the PDFs of Ma and $\chi$ at two

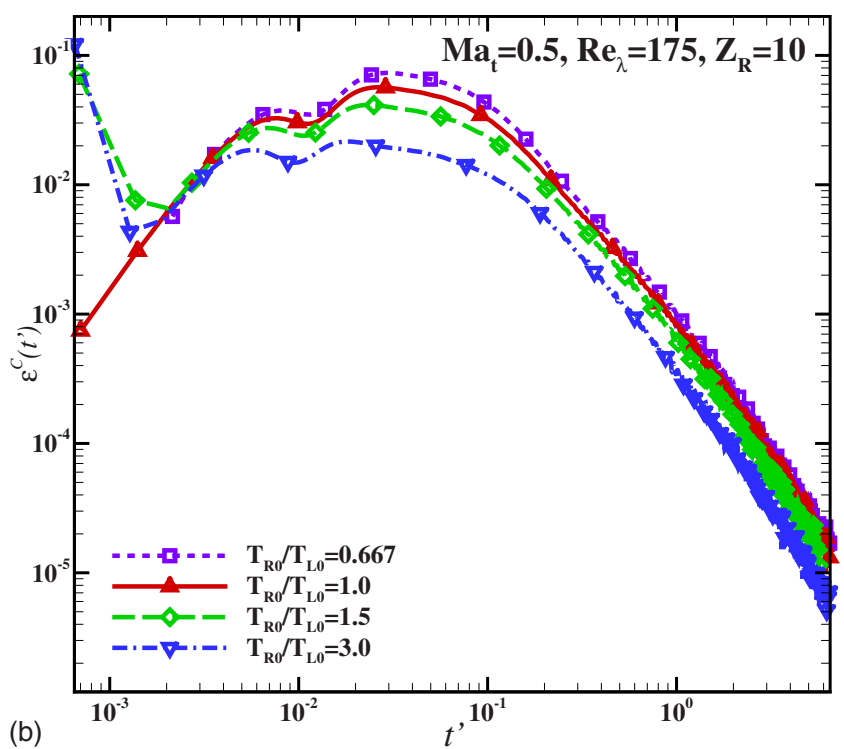

FIG. 17. (Color online) Dependence of the dilatational components of the kinetic energy $K^{C}(t) / K_{0}\left(\right.$ left) and the dissipation rate $\varepsilon^{C}(t) / \varepsilon_{0}$ (right) on the ratio $T_{R 0} / T_{L 0} . \mathrm{Ma}_{t}=0.5, \mathrm{Re}_{\lambda}=175, N^{3}=256^{3}$. 

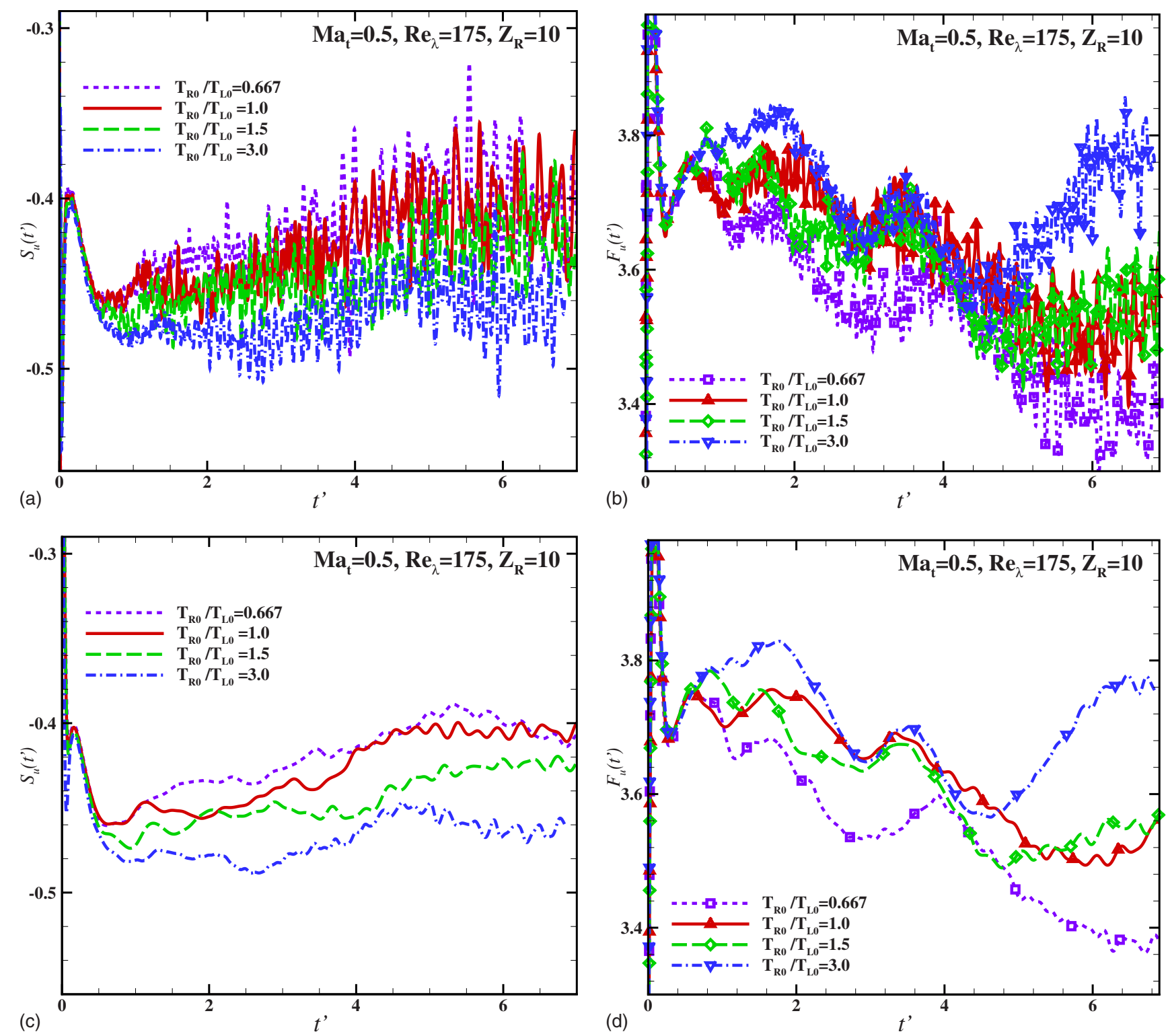

FIG. 18. (Color online) Dependence of the skewness $S_{u}$ (left) and the flatness $F_{u}(t)$ (right) on the ratio $T_{R 0} / T_{L 0} . \mathrm{Ma}_{t}=0.5, \mathrm{Re}_{\lambda}=175$, $N^{3}=256^{3}$. In the lower row, data are smoothed.

times with $\mathrm{Ma}_{t}(0)=0.5, \operatorname{Re}_{\lambda}=72, N^{3}=128^{3}, Z_{R}=10$, and 0.5 $\leq T_{R 0} / T_{L 0} \leq 3.0$. For each value of $T_{R 0} / T_{L 0}$, the first time chosen to output the PDFs is when the dissipation rate $\varepsilon(t) / \varepsilon_{0}$ is about its peak and the second time is $t^{\prime} \approx 5.5$, when the energy decays over 2 orders of magnitude and the turbulence is rather weak already. It should be noted that the initial flow is smooth, so there is no shock. Shocklets will only develop later. However, the shock strength $\chi$ can still be computed for the initial flow field according to Eq. (41a), which is also shown in Fig. 20, and this does not suggest by any means that shocks are present in the initial flow field.

We previously observed that for compressible decaying turbulence, the PDFs of Ma and $\chi$ are self-similar [44]. We rescale the PDFs so that their maximum values are always unity and they are located at 1 . That is, for a PDF $P(x)$, $P(1)=1$ is its maximum. The rescaled PDFs for Ma and $\chi$ are shown in Fig. 21. Clearly, the rescaled PDFs for both the local Mach number Ma and the shocklet strength $\chi$ shown in Fig. 21 are self-similar and they scale as the following:

$$
P(x) \propto e^{\alpha / \beta} x^{\alpha} e^{-\alpha x^{\beta} / \beta}, \quad x \geq 0, \alpha \geq 1, \beta>0,
$$

where $\beta=\alpha=2$ for Ma and $\beta=4 \alpha=4$ for $\chi$. The properly normalized PDF for the local Mach number Ma, i.e., $P(x)=4 x^{2} e^{-x^{2}} / \sqrt{\pi}$, can be derived analytically from the fact that the initial velocity field is Gaussian. However, the properly normalized PDF for the shocklet strength $\chi$, i.e., $P(x)=2 x e^{-x^{4} / 4} / \sqrt{\pi}$, cannot be derived analytically; it is obtained numerically from the data.

In Fig. 22, we show the PDFs of the local Mach number Ma and the shocklet strength $\chi$ under different conditions. Clearly, the rescaled PDFs of both Ma and and $\chi$ are independent of the turbulence Mach number $\mathrm{Ma}_{t}$, the Taylor microscale Reynolds number $\mathrm{Re}_{\lambda}$, and the ratio $T_{R 0} / T_{L 0}$. The 

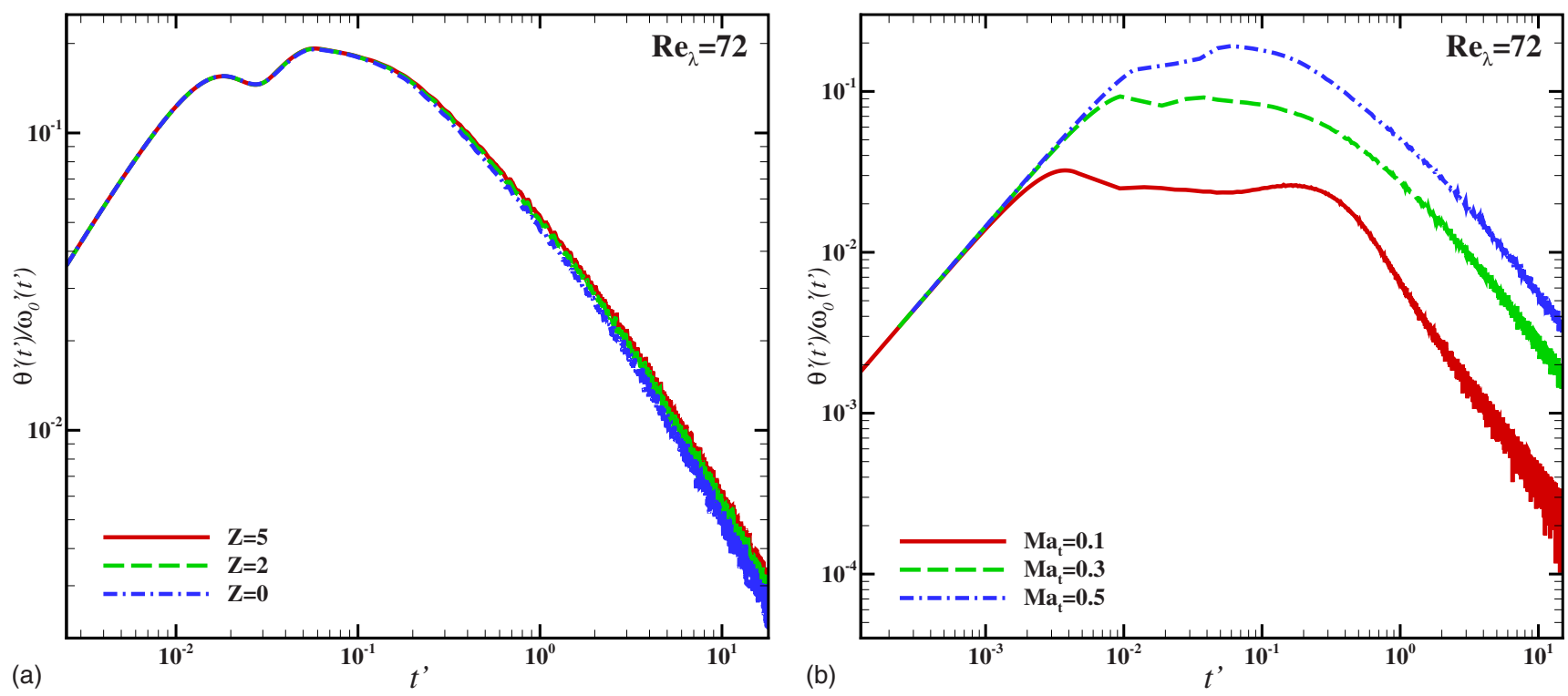

FIG. 19. (Color online) Dependence of the normalized RMS velocity divergence $\theta^{\prime}(t) / \omega_{0}$ on the parameter $Z$ (or the bulk viscosity $\eta$ ) with $\mathrm{Ma}_{t}=0.5$ (left) and the initial turbulence Mach number $\mathrm{Ma}_{t}$ with $Z=2$ (right) for the single-temperature compressible NS equations. $\operatorname{Re}_{\lambda}=72, N^{3}=128^{3}$.

PDFs Ma and $\chi$ are self-similar and they all collapse to the PDFs determined by their initial distributions, which are determined by the initial velocity field. In other words, the self-similarity obeyed by the PDFs appears not to be affected by the details of flow dynamics.

\section{CONCLUSIONS}

In this study, we use the gas-kinetic scheme with the Jeans-Landau-Teller multitemperature model to carry out the direct numerical simulations of the compressible DHIT. We investigate the effects of the multitemperature model on the compressible decaying homogeneous isotropic turbulence by

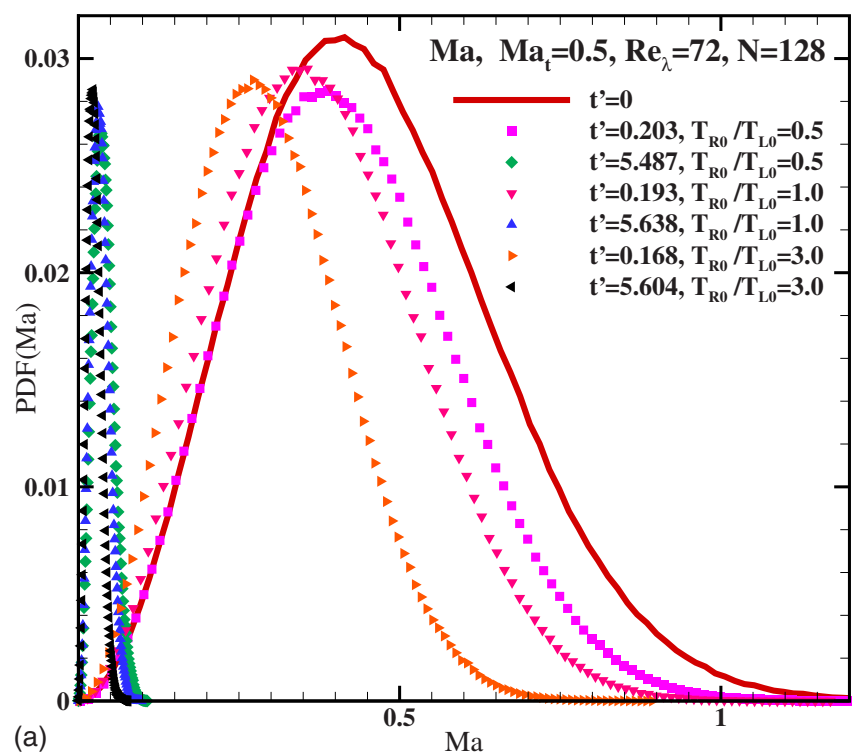

examining the turbulence statistics. In particular, we quantify the effects of the multitemperature model on the total energy $K(t)$ and the dissipation rate $\varepsilon(t)$, as well as their compressible component, the compressible component of the energy spectrum $E^{C}(k, t)$, the skewness $S_{u}(t)$ and the flatness $F_{u}(t)$, and the PDFs of the local Mach number Ma and the shocklet strength $\chi$. Our observations can be summarized as the following.

The multitemperature model is more general than the Navier-Stokes equations with a bulk viscosity and a variable $\gamma$. In the limit of equilibrium, i.e., when the rotational temperature $T_{R}$ is very close to the translational temperature $T_{L}$, the multitemperature model reduces to the compressible

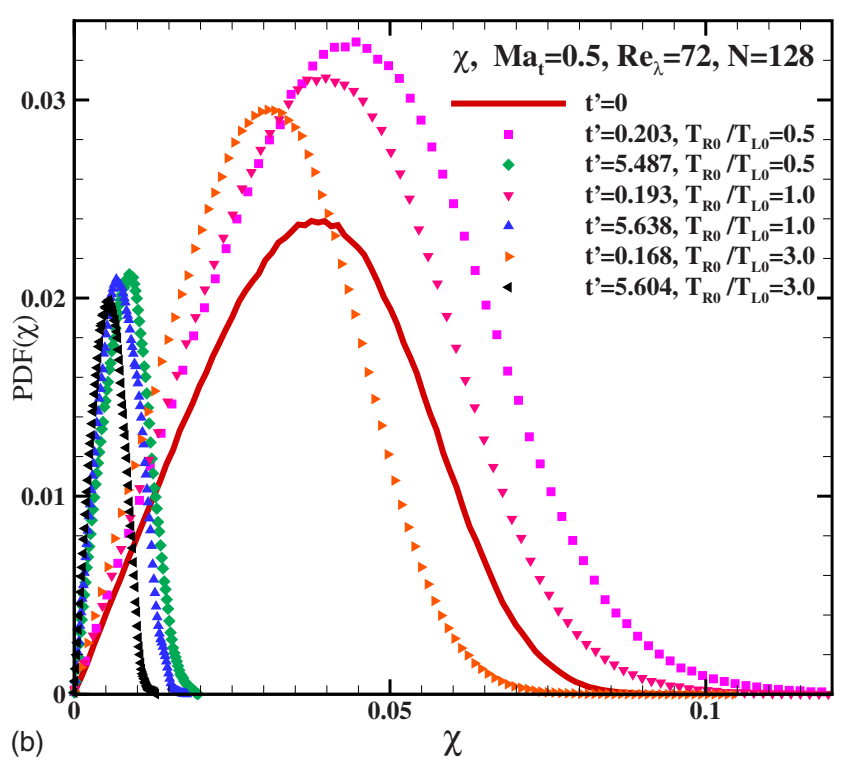

FIG. 20. (Color online) Dependence of PDFs of the local Mach number Ma (left) and the shock strength $\chi$ (right) on $T_{R 0} / T_{L 0}$. $\mathrm{Ma}_{t}=0.5, \operatorname{Re}_{\lambda}=72, N^{3}=128^{3}$. 

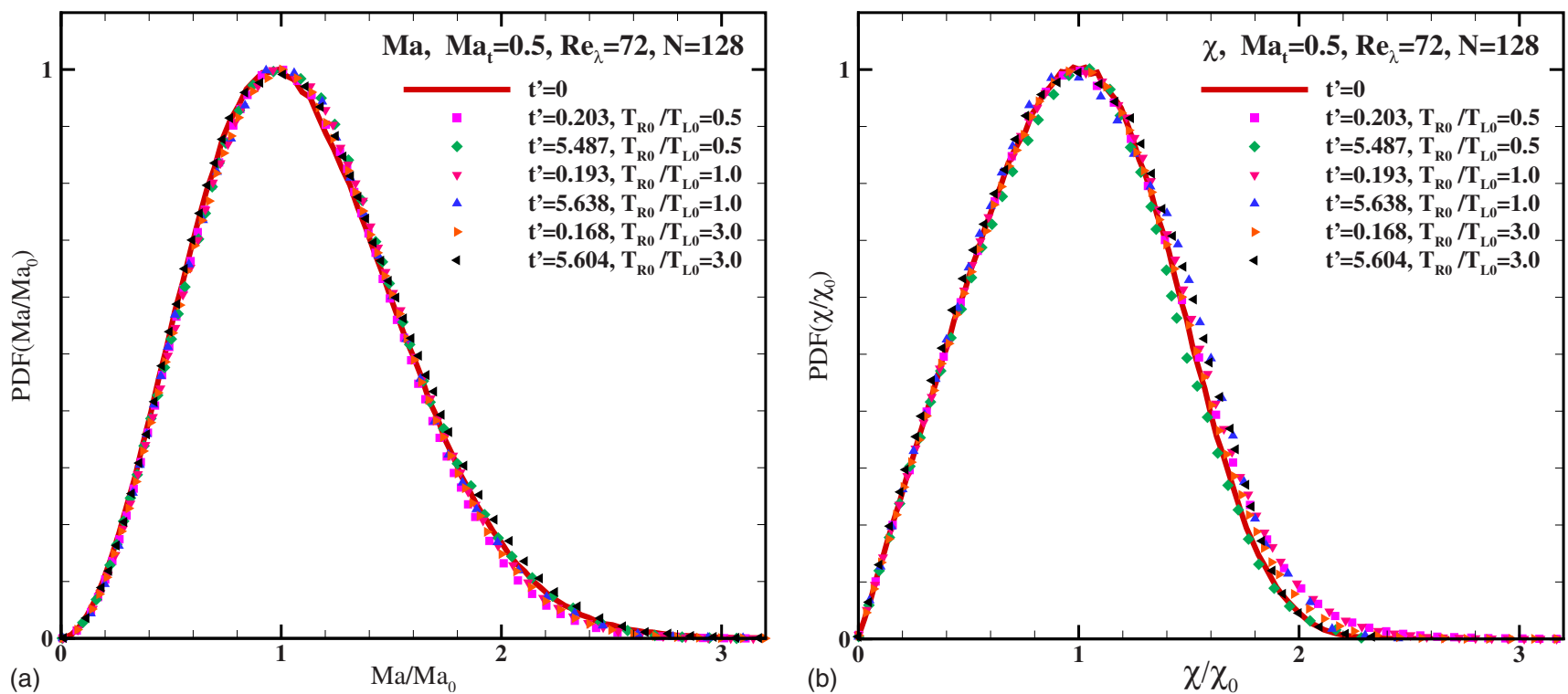

FIG. 21. (Color online) Rescaled PDFs of the local Mach number Ma (left) and the shocklet strength $\chi$ (right) for different $T_{R 0} / T_{L 0}$ with $\mathrm{Ma}_{t}=0.5, \operatorname{Re}_{\lambda}=72$, and $N^{3}=128^{3}$.

Navier-Stokes equations. We note that the relaxation-time scale between the two temperatures $T_{R}$ and $T_{L}$ is rather fast in terms of the turbulence turnover time. We also note that the overall compressibility in a flow is dictated by the Mach number of the flow; thus, the effect of the multitemperature model is only secondary.

The multitemperature model affects the compressibility through the relaxation process of internal degrees of freedom with two adjustable parameters: $Z_{R}$ and $T_{R 0} / T_{L 0}$; the former determines the relaxation time between the two temperatures and the latter determines the initial state. As $Z_{R}$ increases, the rotational temperature relaxes more slowly to its equilibrium and is dissipated more severely in the process, thus the compressibility becomes weaker after a short initial time.
The effect of the parameter $T_{R 0} / T_{L 0}$ is similar, but for a different reason. As $T_{R 0} / T_{L 0}$ increases, so does the equilibrium (translational) temperature $T$ and, hence, the sound speed $c_{s}=\sqrt{\gamma R T}$ after the equilibration process. Consequently, the Mach number $\mathrm{Ma}$ is reduced and the overall compressibility is weakened. Therefore, $T_{R 0}<T_{L 0}$ corresponds to a compressing flow, i.e., the dilatation $\boldsymbol{\nabla} \cdot \boldsymbol{u}$ is enhanced relative to the case of $T_{R 0}=T_{L 0}$, while $T_{R 0}>T_{L 0}$ corresponds to an expanding flow, i.e., the dilatation $\boldsymbol{\nabla} \cdot \boldsymbol{u}$ is suppressed relative to the case of $T_{R 0}=T_{L 0}$. We note that both $Z_{R}$ and $T_{R 0} / T_{L 0}$ affect compressibility and dissipation, but in different ways: the effects of $T_{R 0} / T_{L 0}$ are strong in all times in the decay while the effects of $Z_{R}$ are severe only in the later times passing through the stage with strong nonlinear-

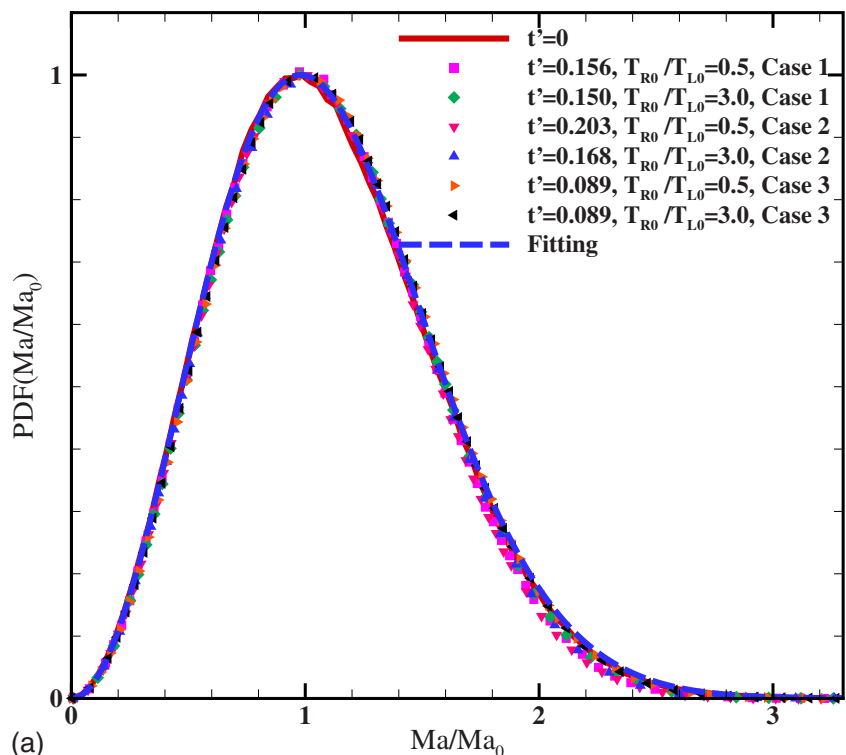

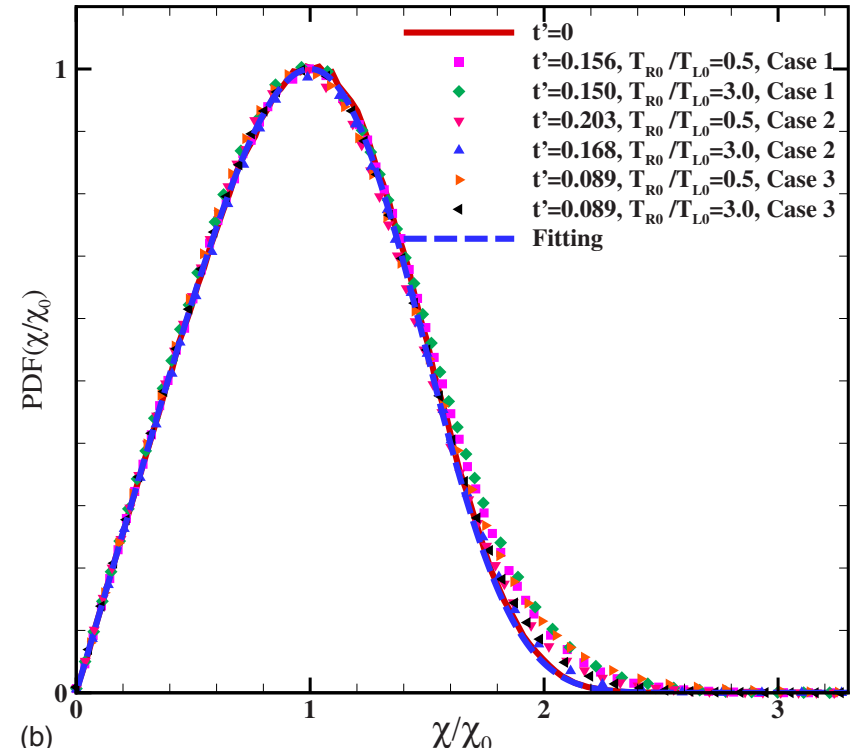

(b)

FIG. 22. (Color online) Rescaled PDFs of the local Mach number Ma (left) and the shocklet strength $\chi$ (right) with different $T_{R 0} / T_{L 0}$ and three initial conditions. Case 1: $\mathrm{Ma}_{t}=0.1, \operatorname{Re}_{\lambda}=72, N^{3}=128^{3} ;$ case 2: $\mathrm{Ma}_{t}=0.5, \operatorname{Re}_{\lambda}=72, N^{3}=128^{3} ;$ case 3: $\operatorname{Ma}_{t}=0.5, \operatorname{Re}_{\lambda}=175, N^{3}=256^{3}$. 
ity. Because the multitemperature model directly affects the compressibility, its effect is more significant for flows with a higher Mach number, in which the compressibility is stronger.

In our previous work on the DNS of the compressible DHIT with a single temperature [44], we discovered that the PDFs of the local Mach number Ma and the shock strength $\chi$ are self-similar. In the present work, we find that the selfsimilarities obeyed by the PDFs of Ma and $\chi$ are not affected by the multitemperature model. This suggests that the self- similarities obeyed by the PDFs of Ma and $\chi$ are a robust feature of the compressible decaying turbulence.

\section{ACKNOWLEDGMENTS}

The authors would like to acknowledge the funding support from U.S. Department of Defense and U.S. Air Force Office of Scientific Research under AFOSR-MURI Project "Hypersonic Transition and Turbulence with NonEquilibrium Thermochemistry."
[1] W. Vincenti and C. Krueger, Introduction to Physical Gas Dynamics (Krieger Wiley, New York, 1965).

[2] J. D. Anderson, Hypersonic and High Temperature Gas Dynamics (McGraw-Hill, New York, 1989). Reprinted by AIAA.

[3] M. El Morsli and P. Proulx, J. Phys. D 40, 4810 (2007).

[4] F. A. Jaberi, D. Livescu, and C. K. Madnia, Phys. Fluids 12, 1189 (2000).

[5] R. Samtaney, D. I. Pullin, and B. Kosović, Phys. Fluids 13, 1415 (2001).

[6] H. Foysi, S. Sarkar, and R. Friedrich, J. Fluid Mech. 509, 207 (2004).

[7] A. E. Honein and P. Moin, J. Comput. Phys. 201(2), 531 (2004).

[8] M. P. Martín and G. V. Candler, J. Comput. Phys. 215, 153 (2006).

[9] M. P. Martín, J. Fluid Mech. 570, 347 (2007).

[10] M. W. Wu and M. P. Martín, J. Fluid Mech. 594, 71 (2008).

[11] M. Ringuette, M. Wu, and M. P. Martín, J. Fluid Mech. 594, 59 (2008).

[12] F. E. Lumpkin, Ph.D. thesis, Stanford University, 1990.

[13] J. H. Jeans. Philos. Mag. 6, 279 (1903).

[14] J. H. Jeans. Philos. Mag. 10, 91 (1905).

[15] L. D. Landau and E. Teller, Phys. Z. Sowjetunion 10, 34 (1936); also in The Collected Papers of L. D. Landau, edited by D. ter Haar (Pergamon, Oxford, 1965), pp. 147-153.

[16] E. E. Nikitin and J. Troe., Phys. Chem. Chem. Phys. 10, 91 (1905).

[17] C. Park, AIAA Paper No. 2010-911 (2010).

[18] F. P. Bertolotti, J. Fluid Mech. 372, 93 (1998).

[19] Yu. N. Grigor'ev, I. V. Ershov, and E. E. Ershova, J. Appl. Mech. Tech. Phys. 45, 321 (2004).

[20] S. Kida and S. A. Orszag, J. Sci. Comput. 5, 1 (1990).

[21] G. A. Blaisdell, N. N. Mansour, and W. C. Reynolds, Stanford University Technical Report No. TF50, 1991 (unpublished).

[22] S. Lee, S. K. Lele, and P. Moin, Phys. Fluids A 3, 657 (1991).

[23] G. Erlebacher, M. Y. Hussaini, C. G. Speziale, and T. A. Zang, J. Fluid Mech. 238, 155 (1992).

[24] W. Liao, Y. Peng, and L.-S. Luo, Phys. Rev. E 80, 046702 (2009).

[25] K. Xu, in The 29th Computational Fluid Dynamics, VKI Lecture Series Vol. 1998-03 (The von Karman Institute for Fluid Dynamics, Rhode-St-Genèse, Belgium, 1998), pp. 1-202.

[26] K. Xu, J. Comput. Phys. 171, 289 (2001).

[27] K. Xu, J. Comput. Phys. 178, 252 (2002).

[28] T. Ohwada, J. Comput. Phys. 177, 156 (2002).

[29] K. Xu, M. L. Mao, and L. Tang, J. Comput. Phys. 203, 405 (2005).
[30] T. Ohwada and S. Fukata, J. Comput. Phys. 211, 424 (2006).

[31] G. May, B. Srinivasan, and A. Jameson, J. Comput. Phys. 220, 856 (2007).

[32] W. Liao, L.-S. Luo, and K. Xu, J. Spacecr. Rockets 44, 1232 (2007).

[33] W. Liao, Y. Peng, L.-S. Luo, and K. Xu, Prog. Comput. Fluid Dyn. 8, 97 (2008).

[34] P. L. Bhatnagar, E. P. Gross, and M. Krook, Phys. Rev. 94, 511 (1954).

[35] K. Xu and Z. H. Li, J. Fluid Mech. 513, 87 (2004).

[36] T. Ohwada and K. Xu, J. Comput. Phys. 201, 315 (2004).

[37] K. Xu and L. Tang, Phys. Fluids 16, 3824 (2004).

[38] K. Xu and E. Josyula, Comm. Comp. Phys. 1, 425 (2006).

[39] K. Xu and E. Josyula, Phys. Rev. E 71, 056308 (2005).

[40] C. P. Cai, D. D. Liu, and K. Xu, AIAA J. 46, 1054 (2008).

[41] K. Xu, H. W. Liu, and J. Z. Jiang, Phys. Fluids 19, 016101 (2007).

[42] K. Xu and H. W. Liu, Comm. Comp. Phys. 4, 1069 (2008).

[43] K. Xu, X. He, and C. P. Cai, J. Comput. Phys. 227, 6779 (2008).

[44] W. Liao, Y. Peng, and L.-S. Luo, AIAA Paper No. 2009-0586 (2009).

[45] W. Liao, Y. Peng, and L.-S. Luo, AIAA Paper No. 2008-0548 (2008).

[46] S. Harris, An Introduction to the Theory of Boltzmann Equation (Holt, Rinehart and Winston, New York, 1971). Reprinted by Dover.

[47] M. N. Kogan, Rarefied Gas Dynamics (Plenum, New York, 1969).

[48] Z. L. Guo, H. W. Liu, L.-S. Luo, and K. Xu, J. Comput. Phys. 227, 4955 (2008).

[49] K. Xu and X. Y. He, J. Comput. Phys. 190, 100 (2003).

[50] C. Park, Nonequilibrium Hypersonic Aerothermodynamics (John Wiley \& Sons, New York, 1990).

[51] L. C. Woods, An Introduction to the Kinetic Theory of Gases and Magnetoplasmas (Oxford University Press, Oxford, UK, 1993).

[52] R. S. Rogallo, NASA Technical Report No. TM-8135, 1981 (unpublished).

[53] T. Passot and A. Pouquet, J. Fluid Mech. 181, 441 (1987).

[54] W. K. Melville, J. Fluid Mech. 51, 571 (1972).

[55] J. A. Lordi and R. E. Mates, Phys. Fluids 13, 291 (1970).

[56] C. Park, AIAA Paper No. 2002-3218 (2002).

[57] S. Tavoularis, J. C. Bennett, and S. Corrsin, J. Fluid Mech. 88(01), 63 (1978)

[58] T. T. Clark and C. Zemarch, Phys. Fluids 10, 2846 (1998). 